\title{
Effects of superimposed eigenstrains on the overall thermoelastic moduli of composites
}

\author{
Djaffar Boussaa \\ boussaa@lma.cnrs-mrs.fr
}

Aix Marseille Univ, CNRS, Centrale Marseille, LMA, Marseille, France

Keywords coefficient of thermal expansion, heat capacity, residual stress, temperature dependent material

\begin{abstract}
This study investigates the effects that an initial local eigenstrain field, when superimposed on the thermal eigenstrain field, has on the overall thermal expansion coefficients and heat capacities of thermoelastic composites. The study can also be seen as an investigation into how a local residual stress field affects these overall moduli, as initial eigenstrains are generally a source of residual stresses. The approach taken is thermodynamic. Expressions that include the superimposed eigenstrain field are developed for the overall moduli within the framework of small strain thermoelasticity with temperature dependent materials. These expressions, which are written in terms of the concentration tensors and residual fields (stress and strain fields given rise to by the eigenstrains under zero overall stress and strain, respectively), contain correction terms that are absent in the expressions developed within linear thermoelasticity. Taking into account the temperature dependence of the constituent moduli is shown to be essential to capture the effects of the superimposed eigenstrain field. A $\mathrm{Ti}-6 \mathrm{Al}-4 \mathrm{~V} / \mathrm{ZrO}_{2}$ composite is investigated for which the correction terms are found to be negligible for the heat capacities but significant for the thermal expansion coefficients. This suggests that, for applications with large temperature changes, using the linear-thermoelasticity-based expressions can affect the accuracy of the estimates of the overall moduli, and therefore the accuracy of thermostructural analyses of composite structures. The proposed expressions can be of use to estimate the overall thermoelastic moduli in contexts in which the strains remain small, temperature changes are large, and superimposed eigenstrains may be present.
\end{abstract}

\section{Introduction}

This study investigates how the thermal expansion coefficients and heat capacities of thermoelastic composites are affected by the presence of an initial local eigenstrain field superimposed on the thermal eigenstrain field. Eigenstrains being generally a source of residual stresses, this study can also be seen as 
an investigation into how local residual stresses affect the overall thermoelastic moduli of composites.

A broader framework than linear thermoelasticity is needed to explore the effects of the superimposed eigenstrain field on the overall thermoelastic moduli. Linear thermoelasticity assumes that the Helmholtz potential is quadratic in strain and temperature (Lubarda, 2004). This assumptions implies, among other things, temperature independent elasticities, which turns out to be too restrictive for the purposes of this study. One such broader framework, and the one used here, is that of small strain thermoelasticity with temperature dependent materials (Kovalenko, 1970). This framework is used in many studies on components having to withstand large temperature changes, including ceramic components under thermal shocks (Han and Wang, 2011; Cheng et al., 2015). For applications of this kind, considering the temperature dependence of the materials is essential in the processes of materials selection (de Faoite et al., 2013), structural analysis (Ching and Chen, 2007), and structural optimization (Bobaru, 2007; Boussaa, 2009).

Being more general, the framework accounts for more phenomena than does linear thermoelasticity. Thus, within the broader framework, Nadeau and Ferrari (2004) showed theoretically that the presence of a superimposed local eigenstrain field can induce anisotropy in the overall thermal expansion response in an otherwise macroscopically isotropic composite of isotropic constituents. They also drew a parallel between their findings and a "most surprising" thermal expansion anisotropy experimentally observed in a polycrystal, one explanation for which could be the presence of residual stresses (Finlayson et al., 1981; Gibbs et al., 1981).

Several approaches have been developed in small strain thermoelasticity to estimate the overall thermoelastic moduli for composites with temperaturedependent constituents. A first approach relies on numerical homogenization. E.g., the finite-element method was used to estimate the overall elasticities and thermal expansion coefficients of silicon nitride (Wippler et al., 2011). A second approach is analytical and yields the overall moduli in terms of the strain and stress concentration tensors and residual fields. One variant of this approach consists in combining the stress-strain relation with the concentration relations (Benveniste and Dvorak, 1990; Bahei-El-Din and Dvorak, 1997; Nadeau and Ferrari, 2004; Dvorak, 2012, pp. 250-252). A second variant consists in combining thermodynamic potentials with the concentration relations (Boussaa, 2011). This second variant has the advantage of providing not only the overall elasticities and thermal expansion coefficients but also the overall heat capacities.

This paper adopts and extends this second variant to accommodate the presence of the superimposed eigenstrain field, and assesses the effects of that presence on the overall thermal expansion coefficients as well as on the overall heat capacities.

The paper is organized as follows. Section 2 provides notation and the definitions of the material moduli of interest here. Section 3 develops expressions for the Gibbs and Helmholtz potentials of a homogeneous material in which an initial eigenstrain is superimposed on the thermal eigenstrain. Section 4 recalls the concentration relations and obtains the macroscopic Gibbs and Helmholtz potentials of a heterogeneous material with an initial superimposed eigenstrain field. Section 5 derives the overall moduli from the macroscopic thermody- 
namic potentials. Section 6 provides Levin-type formulas for two-phase composites. Section 7 discusses a numerical example illustrating the application of the developed framework to estimate the overall moduli of a high temperature composite with a superimposed eigenstrain field. Finally, Section 8 gives some concluding remarks.

\section{Thermoelastic material moduli of interest}

The material moduli of interest here are the isothermal elasticity tensor, $\boldsymbol{L}$; the isothermal compliance tensor, $\boldsymbol{M}$; the coefficient of thermal expansion (CTE) tensor, $\boldsymbol{\alpha}$; the stress-temperature tensor, $\boldsymbol{\beta}$; the heat capacity per unit reference volume at constant strain, $C_{\epsilon}$; and the heat capacity per unit reference volume at constant stress, $C_{\boldsymbol{\sigma}}$. These moduli are defined as follows:

$$
\begin{aligned}
\boldsymbol{L} & =\left(\frac{\partial \boldsymbol{\sigma}}{\partial \boldsymbol{\epsilon}}\right)_{T}, & \boldsymbol{M} & =\left(\frac{\partial \boldsymbol{\epsilon}}{\partial \boldsymbol{\sigma}}\right)_{T}, \\
\boldsymbol{\alpha} & =\left(\frac{\partial \boldsymbol{\epsilon}}{\partial T}\right)_{\boldsymbol{\sigma}}, & \boldsymbol{\beta} & =\left(\frac{\partial \boldsymbol{\sigma}}{\partial T}\right)_{\boldsymbol{\epsilon}}, \\
C_{\boldsymbol{\epsilon}} & =T\left(\frac{\partial \eta}{\partial T}\right)_{\boldsymbol{\epsilon}}, & C_{\boldsymbol{\sigma}} & =T\left(\frac{\partial \eta}{\partial T}\right)_{\boldsymbol{\sigma}},
\end{aligned}
$$

where $\boldsymbol{\sigma}$ is the stress tensor, $\boldsymbol{\epsilon}$ is the small strain tensor, $T$ is the absolute temperature, and $\eta$ is the entropy.

The moduli thus defined depend a priori on the two independent variables used to describe the thermodynamic state of the material, which here are taken to be either stress and temperature or strain and temperature. When stress and temperature are used as independent variables, the dual variables, strain and entropy, are given by

$$
\boldsymbol{\epsilon}=-\left(\frac{\partial g}{\partial \boldsymbol{\sigma}}\right)_{T}, \quad \eta=-\left(\frac{\partial g}{\partial T}\right)_{\boldsymbol{\sigma}},
$$

where $g$ is the Gibbs potential per unit reference volume. Similarly, when strain and temperature are used as independent variables, the dual variables, stress and entropy, are given by

$$
\boldsymbol{\sigma}=\left(\frac{\partial f}{\partial \boldsymbol{\epsilon}}\right)_{T}, \quad \eta=-\left(\frac{\partial f}{\partial T}\right)_{\boldsymbol{\epsilon}},
$$

where $f$ is the Helmholtz potential per unit reference volume.

From the above definitions, it follows that the moduli enjoy the usual symmetries.

The symbols $f_{0}, g_{0}$ and $\eta_{0}$ will be used to denote the values of $f, g$, and $\eta$ at some reference state, respectively. The reference state referred to will be specified at each use. The dot "." will denote the usual inner product between second-order tensors: For any two second-order tensors $\boldsymbol{X}$ and $\boldsymbol{Y}, \boldsymbol{X} \cdot \boldsymbol{Y}=$ $X_{i j} Y_{i j}$, with summation over repeated indices.

The overall or effective moduli will be denoted with superscript "eff". 


\section{Gibbs and Helmholtz potentials of a homoge- neous thermoelastic material with a superim- posed eigenstrain}

In (Boussaa, 2011), which deals with the case without superimposed eigenstrain, the starting point for developing expressions for the Gibbs and Helmholtz potentials is the postulation of appropriate forms for them. Here, it is more straightforward to start with the postulation of constitutive relations, and then integrate them to obtain expressions for the potentials. The constitutive relations are

$$
\begin{aligned}
\boldsymbol{\epsilon} & =\boldsymbol{M}(T) \boldsymbol{\sigma}+\boldsymbol{m}(T), \\
\boldsymbol{m}(T) & =\boldsymbol{m}_{\mathrm{th}}(T)+\boldsymbol{m}_{*},
\end{aligned}
$$

where $\boldsymbol{m}$ is the total eigenstrain, $\boldsymbol{m}_{\mathrm{th}}$ is the thermal eigenstrain, and $\boldsymbol{m}_{*}$ is the superimposed eigenstrain, which is assumed not to depend on $T$ and whose physical origin is irrelevant to the analysis. The linear stress-strain relationship (6), the additive decomposition (7), and the compliance tensor dependence on the temperature but not on the superimposed eigenstrain are the core assumptions of the constitutive model. These are common assumptions in the framework of small strains.

\subsection{Gibbs potential}

In view of the stress-strain relation (6), integrating (4-1) gives

$$
g(\boldsymbol{\sigma}, T)=-\frac{1}{2} \boldsymbol{\sigma} \cdot \boldsymbol{M}(T) \boldsymbol{\sigma}-\boldsymbol{m}(T) \cdot \boldsymbol{\sigma}+\phi(T),
$$

where $\phi$ is a function of the temperature alone. From (3-2), (4-2) and (8), one can relate $\phi$ to $C_{\boldsymbol{\sigma}}$ as follows:

$$
C_{\boldsymbol{\sigma}}(\boldsymbol{\sigma}, T)=T\left(\frac{1}{2} \boldsymbol{\sigma} \cdot \frac{d^{2} \boldsymbol{M}}{d T^{2}} \boldsymbol{\sigma}+\frac{d^{2} \boldsymbol{m}}{d T^{2}} \cdot \boldsymbol{\sigma}-\frac{d^{2} \phi}{d T^{2}}\right) .
$$

Writing this equation for $\boldsymbol{\sigma}=\mathbf{0}$, dividing both sides by $T$, and integrating the resulting equation twice with respect to temperature gives

$$
\begin{aligned}
\phi(T)= & -\int_{T_{0}}^{T}\left(\int_{T_{0}}^{\xi} C_{\boldsymbol{\sigma}}(\mathbf{0}, \nu) \frac{d \nu}{\nu}\right) d \xi \\
& -\left(T-T_{0}\right) \eta_{0}+g_{0},
\end{aligned}
$$

where $T_{0}$ is an arbitrary temperature, and $\eta_{0}$ and $g_{0}$ are the entropy and the Gibbs potential in the state characterized by $\boldsymbol{\sigma}=\mathbf{0}$ and $T=T_{0}$, respectively.

Substituting (10) back into (8) gives the desired expression for the Gibbs potential:

$$
\begin{aligned}
g(\boldsymbol{\sigma}, T)= & -\frac{1}{2} \boldsymbol{\sigma} \cdot \boldsymbol{M}(T) \boldsymbol{\sigma}-\boldsymbol{m}(T) \cdot \boldsymbol{\sigma} \\
& -\int_{T_{0}}^{T}\left(\int_{T_{0}}^{\xi} C_{\boldsymbol{\sigma}}(\mathbf{0}, \nu) \frac{d \nu}{\nu}\right) d \xi
\end{aligned}
$$




$$
-\left(T-T_{0}\right) \eta_{0}+g_{0} .
$$

The following remarks about the thermoelastic constitutive model characterized by this potential are in order:

1. The superimposed eigenstrain does not affect the compliance (by assumption).

2. By (2-1) and (4-2), the CTE tensor is given by

$$
\boldsymbol{\alpha}(\boldsymbol{\sigma}, T)=\frac{d \boldsymbol{M}}{d T} \boldsymbol{\sigma}+\frac{d \boldsymbol{m}_{\mathrm{th}}}{d T} .
$$

This equation shows that the CTE tensor is independent of the superimposed eigenstrain, $\boldsymbol{m}_{*}$, and that

$$
\boldsymbol{m}_{\mathrm{th}}(T)=\int_{T_{\mathrm{ref}}}^{T} \boldsymbol{\alpha}(\mathbf{0}, \nu) \mathrm{d} \nu,
$$

where $T_{\text {ref }}$ is the temperature for which the thermal eigenstrain vanishes (i.e., $\boldsymbol{m}_{\mathrm{th}}\left(T_{\text {ref }}\right)=0$ ). The quantity $\boldsymbol{\alpha}(\mathbf{0}, T)$ is the coefficient of free thermal expansion (CFTE) tensor. It is related to $\boldsymbol{\alpha}(\boldsymbol{\sigma}, T)$ through

$$
\boldsymbol{\alpha}(\boldsymbol{\sigma}, T)=\frac{d \boldsymbol{M}}{d T} \boldsymbol{\sigma}+\boldsymbol{\alpha}(\mathbf{0}, T) .
$$

3. By (3-2), the heat capacity at constant stress is

$$
\begin{aligned}
C_{\boldsymbol{\sigma}}(\boldsymbol{\sigma}, T)= & C_{\boldsymbol{\sigma}}(\mathbf{0}, T) \\
& +T\left(\frac{1}{2} \boldsymbol{\sigma} \cdot \frac{d^{2} \boldsymbol{M}}{d T^{2}} \boldsymbol{\sigma}+\frac{d^{2} \boldsymbol{m}_{\mathrm{th}}}{d^{2} T^{2}} \cdot \boldsymbol{\sigma}\right) .
\end{aligned}
$$

This shows that the heat capacity at constant stress also is independent of the superimposed eigenstrain, $\boldsymbol{m}_{*}$.

4. The choice of $\boldsymbol{\sigma}=\mathbf{0}$ as a reference stress in deriving (11) is one of convenience, as it leads to a compact expression for the Gibbs potential. Any choice of this reference stress, however, is equally valid. Writing (9) for $\boldsymbol{\sigma}=\boldsymbol{\sigma}_{0}$, where $\boldsymbol{\sigma}_{0}$ is arbitrary, and proceeding as was done for $\boldsymbol{\sigma}=\mathbf{0}$ gives

$$
\begin{aligned}
g(\boldsymbol{\sigma}, T)= & -\frac{1}{2} \boldsymbol{\sigma} \cdot \boldsymbol{M}(T) \boldsymbol{\sigma}-\boldsymbol{m}(T) \cdot \boldsymbol{\sigma} \\
& +\frac{1}{2} \boldsymbol{\sigma}_{0} \cdot \boldsymbol{M}(T) \boldsymbol{\sigma}_{0}+\boldsymbol{m}(T) \cdot \boldsymbol{\sigma}_{0} \\
& -\int_{T_{0}}^{T}\left(\int_{T_{0}}^{\xi} C_{\boldsymbol{\sigma}}\left(\boldsymbol{\sigma}_{0}, \nu\right) \frac{d \nu}{\nu}\right) d \xi \\
& -\left(T-T_{0}\right) \eta_{0}+g_{0},
\end{aligned}
$$

where $\eta_{0}$ and $g_{0}$ are here the entropy and the Gibbs potential in the state characterized by $\boldsymbol{\sigma}=\boldsymbol{\sigma}_{\mathbf{0}}$ and $T=T_{0}$, respectively. 
5. In summary, within the present thermoelastic framework, the material data needed to specify the Gibbs potential of a homogeneous material, and thus its state equations and moduli at any state, are the following: $\boldsymbol{M}(T)$, the value of the compliance tensor as a function of temperature; $\boldsymbol{m}_{\mathrm{th}}$, the value of the thermal eigenstrain as a function of temperature; $\boldsymbol{m}_{*}$, the value of the superimposed eigenstrain; $C_{\boldsymbol{\sigma}}\left(\boldsymbol{\sigma}_{0}, T\right)$, the value the heat capacity at constant stress as a function of temperature at an arbitrary given value of the stress $\boldsymbol{\sigma}_{0}$; and $\eta_{0}$ and $g_{0}$, the values of the entropy and Gibbs potential at a reference state $\left(\sigma_{0}, T_{0}\right)$, respectively. The quantities $\eta_{0}$ and $g_{0}$ do not enter the expressions for the moduli.

\subsection{Helmholtz potential}

The Helmholtz potential, $f$, is obtained by Legendre transforming expression

(11) for the Gibbs potential with respect to stress, that is,

$$
f=g+\boldsymbol{\sigma} \cdot \boldsymbol{\epsilon}
$$

Inverting the stress-strain relation (6) and combining the resulting equation with (15) and definition (3-1) of $C_{\boldsymbol{\epsilon}}$ gives

$$
\begin{aligned}
f(\boldsymbol{\epsilon}, T)= & \frac{1}{2} \boldsymbol{\epsilon} \cdot \boldsymbol{L}(T) \boldsymbol{\epsilon}+\boldsymbol{l}(T) \cdot \boldsymbol{\epsilon} \\
& -\int_{T_{0}}^{T}\left(\int_{T_{0}}^{\xi} C_{\boldsymbol{\epsilon}}(\mathbf{0}, \nu) \frac{d \nu}{\nu}\right) d \xi \\
& -\left(T-T_{0}\right) \eta_{0}+f_{0},
\end{aligned}
$$

where $\eta_{0}$ and $f_{0}$ are here the entropy and the Helmholtz potential in the state characterized by $\boldsymbol{\epsilon}=\mathbf{0}$ and $T=T_{0}$, respectively, and the total eigenstress, $\boldsymbol{l}$, is defined by

$$
\boldsymbol{l}(T)=-\boldsymbol{L}(T) \boldsymbol{m}(T) .
$$

The state equations associated with the Helmholtz potential (16) read

$$
\begin{aligned}
\boldsymbol{\sigma}= & \boldsymbol{L} \boldsymbol{\epsilon}+\boldsymbol{l}, \\
\eta= & -\frac{1}{2} \boldsymbol{\epsilon} \cdot \frac{d \boldsymbol{L}}{d T} \boldsymbol{\epsilon}-\frac{d \boldsymbol{l}}{d T} \cdot \boldsymbol{\epsilon} \\
& +\int_{T_{0}}^{T} C_{\boldsymbol{\epsilon}}(\mathbf{0}, \nu) \frac{d \nu}{\nu}+\eta_{0} .
\end{aligned}
$$

The following remarks complement those in the previous subsection:

1. From the state equation for stress (18), the stress-temperature tensor $\boldsymbol{\beta}(\boldsymbol{\epsilon}, T)$ is given by

$$
\boldsymbol{\beta}(\boldsymbol{\epsilon}, T)=\frac{d \boldsymbol{L}}{d T} \boldsymbol{\epsilon}+\frac{d \boldsymbol{l}}{d T} .
$$

In view of definition (17) of $\boldsymbol{l}$, the stress-temperature tensor $\boldsymbol{\beta}(\boldsymbol{\epsilon}, T)$ depends on the superimposed eigenstrain, unless $\boldsymbol{L}$ is independent of $T$.

2. The state equation for entropy (19) implies that

$$
C_{\boldsymbol{\epsilon}}(\boldsymbol{\epsilon}, T)=C_{\boldsymbol{\epsilon}}(\mathbf{0}, T)
$$




$$
-T\left(\frac{1}{2} \boldsymbol{\epsilon} \cdot \frac{d^{2} \boldsymbol{L}}{d T^{2}} \boldsymbol{\epsilon}+\frac{d^{2} \boldsymbol{l}}{d T^{2}} \cdot \boldsymbol{\epsilon}\right) .
$$

Again, unless $\boldsymbol{L}$ is independent of, or varies linearly with, $T$, the heat capacity $C_{\boldsymbol{\epsilon}}(\boldsymbol{\epsilon}, T)$ depends on the superimposed eigenstrain.

3. The reference strain $\boldsymbol{\epsilon}=\mathbf{0}$ used to express $f$ is again a choice of convenience. The expression for the Helmholtz potential for an arbitrary reference state $\left(\boldsymbol{\epsilon}=\boldsymbol{\epsilon}_{0}, T=T_{0}\right)$ is given by

$$
\begin{aligned}
f(\boldsymbol{\epsilon}, T)= & \frac{1}{2} \boldsymbol{\epsilon} \cdot \boldsymbol{L}(T) \boldsymbol{\epsilon}+\boldsymbol{l}(T) \cdot \boldsymbol{\epsilon} \\
& -\frac{1}{2} \boldsymbol{\epsilon}_{0} \cdot \boldsymbol{L}(T) \boldsymbol{\epsilon}_{0}-\boldsymbol{l}(T) \cdot \boldsymbol{\epsilon}_{0} \\
& -\int_{T_{0}}^{T}\left(\int_{T_{0}}^{\xi} C_{\boldsymbol{\epsilon}}\left(\boldsymbol{\epsilon}_{0}, \nu\right) \frac{d \nu}{\nu}\right) d \xi \\
& -\left(T-T_{0}\right) \eta_{0}+f_{0},
\end{aligned}
$$

where $\eta_{0}$ and $g_{0}$ are here the entropy and the Helmholtz potential in the reference state $\left(\boldsymbol{\epsilon}=\boldsymbol{\epsilon}_{0}, T=T_{0}\right)$, respectively.

\section{Gibbs and Helmholtz potentials of a heteroge- neous thermoelastic material with a superim- posed eigenstrain field}

\subsection{Assumptions and concentration relations}

A representative volume element (RVE) of a heterogeneous material is considered. Its constituents are assumed to show (i) a thermoelastic behavior governed by the Gibbs potential (11) or (14), or equivalently by the Helmholtz potential (16) or (21), and the associated state equations, and (ii) perfect bonding and thermal contact at their interfaces. Within the RVE, (iii) the superimposed eigenstrain field is assumed to be given, (iv) the temperature is assumed to be uniform, and (v) the reference temperature for zero thermal strain is the same for all constituents.

If the RVE is considered under controlled macroscopic stress $\bar{\sigma}$ and temperature $T$, then the local stress $\boldsymbol{\sigma}(\boldsymbol{x})$ can be written as

$$
\boldsymbol{\sigma}(\boldsymbol{x})=\boldsymbol{B}(\boldsymbol{x} ; T) \overline{\boldsymbol{\sigma}}+\boldsymbol{b}(\boldsymbol{x} ; T) .
$$

In this equation, the term $\boldsymbol{B} \overline{\boldsymbol{\sigma}}$ is the stress that develops in the RVE under $\overline{\boldsymbol{\sigma}}$ alone, and the fourth-order tensor $\boldsymbol{B}$, which is independent of $\boldsymbol{m}_{*}$, is the stress concentration tensor at temperature $T$. The residual stress $\boldsymbol{b}$ is the stress that develops in the RVE under the total eigenstrain field $\boldsymbol{m}$ and zero overall stress conditions. By linearity, $\boldsymbol{b}$ can be decomposed into two terms,

$$
\boldsymbol{b}(\boldsymbol{x} ; T)=\boldsymbol{b}_{\mathrm{th}}(\boldsymbol{x} ; T)+\boldsymbol{b}_{*}(\boldsymbol{x} ; T),
$$

the first of which represents the stress developed under $\boldsymbol{m}_{\mathrm{th}}(\boldsymbol{x} ; T)$ alone while the second represents the stress developed under $\boldsymbol{m}_{*}(\boldsymbol{x})$ alone. Although the 
stress $\boldsymbol{b}_{*}(\boldsymbol{x} ; T)$ arises from the temperature-independent superimposed eigenstrain, $\boldsymbol{m}_{*}$, it generally depends on the temperature, the elasticity tensors of the constituents being temperature dependent. In the case of temperature independent constituent elasticities and CFTEs, $\boldsymbol{m}$ is affine in $T$, and so is $\boldsymbol{b}$, by linearity again. If either the elasticities or CFTEs or both depend on temperature, then $\boldsymbol{b}$ is, in general, non affine in $T$.

If the RVE is considered under controlled macroscopic strain $\bar{\epsilon}$ and temperature $T$, then the local strain $\boldsymbol{\epsilon}(\boldsymbol{x})$ can be written as

$$
\boldsymbol{\epsilon}(\boldsymbol{x})=\boldsymbol{A}(\boldsymbol{x} ; T) \overline{\boldsymbol{\epsilon}}+\boldsymbol{a}(\boldsymbol{x} ; T) .
$$

In this equation, the term $\boldsymbol{A} \overline{\boldsymbol{\epsilon}}$ is the strain that develops in the RVE under $\overline{\boldsymbol{\epsilon}}$ alone, and the fourth-order tensor $\boldsymbol{A}$, which is independent of $\boldsymbol{m}_{*}$, is the strain concentration tensor at temperature $T$. The residual strain $\boldsymbol{a}$ is the strain that develops in the RVE under the eigenstrains and zero overall strain conditions. By linearity again, $\boldsymbol{a}$ also can be decomposed into two terms,

$$
\boldsymbol{a}(\boldsymbol{x} ; T)=\boldsymbol{a}_{\mathrm{th}}(\boldsymbol{x} ; T)+\boldsymbol{a}_{*}(\boldsymbol{x} ; T),
$$

the first of which represents the strain developed under $\boldsymbol{m}_{\text {th }}(\boldsymbol{x} ; T)$ alone while the second represents the strain developed under $\boldsymbol{m}_{*}(\boldsymbol{x})$ alone. Although the strain $\boldsymbol{a}_{*}(\boldsymbol{x} ; T)$ arises from eigenstrain $\boldsymbol{m}_{*}$, it depends on the temperature because of the temperature dependence of the constituent elasticities.

The terminology "residual stress" and "residual strain" is adopted from (Dvorak, 2012, p. 54).

\subsection{Macroscopic Gibbs potential}

The macroscopic Gibbs potential density $G$ is defined as

$$
G(\overline{\boldsymbol{\sigma}}, T)=\langle g\rangle=\frac{1}{|V|} \int_{V} g(\boldsymbol{x} ; \boldsymbol{\sigma}, T) \mathrm{d} V .
$$

Here, $g(\boldsymbol{x} ; \boldsymbol{\sigma}, T)$ is of the form (11) (or 14) where $\boldsymbol{M}, \boldsymbol{m}, C_{\boldsymbol{\sigma}}, \eta_{0}$, and $g_{0}$, and $T_{0}$ are made to depend on position, and $\boldsymbol{\sigma}$ is the stress that develops in the RVE under the combined effects of $\overline{\boldsymbol{\sigma}}$ and $\boldsymbol{m}, V$ is an RVE and $|V|$ its volume, and $\langle\cdot\rangle$ is the average over $V$.

Substituting expression (11) for $g$ and the stress concentration relation (22) into the foregoing equation gives

$$
\begin{aligned}
G(\overline{\boldsymbol{\sigma}}, T)= & -\frac{1}{2} \overline{\boldsymbol{\sigma}} \cdot\left\langle\boldsymbol{B}^{\boldsymbol{\top}} \boldsymbol{M} \boldsymbol{B}\right\rangle \overline{\boldsymbol{\sigma}} \\
& -\left\langle\boldsymbol{B}^{\boldsymbol{\top}}(\boldsymbol{M} \boldsymbol{b}+\boldsymbol{m})\right\rangle \cdot \overline{\boldsymbol{\sigma}} \\
& -\left\langle\frac{1}{2} \boldsymbol{b} \cdot \boldsymbol{M} \boldsymbol{b}+\boldsymbol{m} \cdot \boldsymbol{b}\right\rangle \\
& -\left\langle\int_{T_{0}}^{T}\left(\int_{T_{0}}^{\xi} C_{\boldsymbol{\sigma}}(\mathbf{0}, \nu) \frac{d \nu}{\nu}\right) d \xi\right\rangle \\
& -\left\langle\left(T-T_{0}\right) \eta_{0}\right\rangle+\left\langle g_{0}\right\rangle .
\end{aligned}
$$

The derivation of this equation uses classical properties of the stress concentration tensor $\boldsymbol{B}$ and residual stress $\boldsymbol{b}$, as can be found in (Boussaa, 2011). 
If the reference state is $\left(\boldsymbol{\sigma}_{0}(\boldsymbol{x}), T_{0}(\boldsymbol{x})\right)$, then the expression for $G$ takes the following form:

$$
\begin{aligned}
G(\overline{\boldsymbol{\sigma}}, T)= & -\frac{1}{2} \overline{\boldsymbol{\sigma}} \cdot\left\langle\boldsymbol{B}^{\boldsymbol{\top}} \boldsymbol{M} \boldsymbol{B}\right\rangle \overline{\boldsymbol{\sigma}} \\
& -\left\langle\boldsymbol{B}^{\boldsymbol{\top}}(\boldsymbol{M} \boldsymbol{b}+\boldsymbol{m})\right\rangle \cdot \overline{\boldsymbol{\sigma}} \\
& -\left\langle\frac{1}{2} \boldsymbol{b} \cdot \boldsymbol{M} \boldsymbol{b}+\boldsymbol{m} \cdot \boldsymbol{b}\right\rangle \\
& +\frac{1}{2}\left\langle\boldsymbol{\sigma}_{0} \cdot \boldsymbol{M} \boldsymbol{\sigma}_{0}\right\rangle+\left\langle\boldsymbol{\sigma}_{0} \cdot \boldsymbol{m}\right\rangle \\
& -\left\langle\int_{T_{0}}^{T}\left(\int_{T_{0}}^{\xi} C_{\boldsymbol{\sigma}}\left(\boldsymbol{\sigma}_{0}, \nu\right) \frac{d \nu}{\nu}\right) d \xi\right\rangle \\
& -\left\langle\left(T-T_{0}\right) \eta_{0}\right\rangle+\left\langle g_{0}\right\rangle .
\end{aligned}
$$

\subsection{Macroscopic Helmholtz potential}

Similarly to the macroscopic Gibbs potential, the macroscopic Helmholtz potential density $F$ is defined as

$$
F(\overline{\boldsymbol{\epsilon}}, T)=\langle f\rangle=\frac{1}{|V|} \int_{V} f(\boldsymbol{x} ; \boldsymbol{\epsilon}, T) \mathrm{d} V .
$$

Here, $f(\boldsymbol{x} ; \boldsymbol{\sigma}, T)$ is of the form (16) where $\boldsymbol{L}, \boldsymbol{m}, C_{\boldsymbol{\epsilon}}, \eta_{0}, f_{0}$, and $T_{0}$ are made to depend on position, and $\epsilon$ is the strain that develops in the RVE under the combined effects of $\overline{\boldsymbol{\epsilon}}$ and $\boldsymbol{m}$.

Substituting expression (16) for $f$ and the strain concentration relation (23) into the foregoing equation gives

$$
\begin{aligned}
F(\overline{\boldsymbol{\epsilon}}, T)= & \frac{1}{2} \overline{\boldsymbol{\epsilon}} \cdot\left\langle\boldsymbol{A}^{\boldsymbol{\top}} \boldsymbol{L} \boldsymbol{A}\right\rangle \overline{\boldsymbol{\epsilon}} \\
& +\left\langle\boldsymbol{A}^{\boldsymbol{\top}}(\boldsymbol{L} \boldsymbol{a}+\boldsymbol{l})\right\rangle \cdot \overline{\boldsymbol{\epsilon}} \\
& +\left\langle\frac{1}{2} \boldsymbol{a} \cdot \boldsymbol{L} \boldsymbol{a}+\boldsymbol{l} \cdot \boldsymbol{a}\right\rangle \\
& -\left\langle\int_{T_{0}}^{T}\left(\int_{T_{0}}^{\xi} C_{\boldsymbol{\epsilon}}(\mathbf{0}, \nu) \frac{d \nu}{\nu}\right) d \xi\right\rangle \\
& -\left\langle\left(T-T_{0}\right) \eta_{0}\right\rangle+\left\langle f_{0}\right\rangle .
\end{aligned}
$$

Again, the derivation of this equation uses classical properties of tensors $\boldsymbol{A}$ and $\boldsymbol{a}$, as can be found in (Boussaa, 2011).

If a state $\left(\boldsymbol{\epsilon}_{0}(\boldsymbol{x}), T_{0}(\boldsymbol{x})\right)$ is taken as the reference state instead of $\left(\mathbf{0}, T_{0}(\boldsymbol{x})\right)$, then the expression for $F$ becomes

$$
\begin{aligned}
F(\overline{\boldsymbol{\epsilon}}, T)= & \frac{1}{2} \overline{\boldsymbol{\epsilon}} \cdot\left\langle\boldsymbol{A}^{\top} \boldsymbol{L} \boldsymbol{A}\right\rangle \overline{\boldsymbol{\epsilon}} \\
& +\left\langle\boldsymbol{A}^{\top} \boldsymbol{L}(\boldsymbol{a}-\boldsymbol{m})\right\rangle \cdot \overline{\boldsymbol{\epsilon}} \\
& +\left\langle\frac{1}{2} \boldsymbol{a} \cdot \boldsymbol{L} \boldsymbol{a}-\boldsymbol{a} \cdot \boldsymbol{L} \boldsymbol{m}\right\rangle \\
& -\frac{1}{2}\left\langle\boldsymbol{\epsilon}_{0} \cdot \boldsymbol{L} \boldsymbol{\epsilon}_{0}\right\rangle-\left\langle\boldsymbol{\epsilon}_{0} \cdot \boldsymbol{L} \boldsymbol{m}\right\rangle
\end{aligned}
$$




$$
\begin{aligned}
& -\left\langle\int_{T_{0}}^{T}\left(\int_{T_{0}}^{\xi} C_{\boldsymbol{\epsilon}}\left(\boldsymbol{\epsilon}_{0}, \nu\right) \frac{d \nu}{\nu}\right) d \xi\right\rangle \\
& -\left\langle\left(T-T_{0}\right) \eta_{0}\right\rangle+\left\langle f_{0}\right\rangle .
\end{aligned}
$$

\section{Overall moduli}

\subsection{Values deduced from the macroscopic Gibbs potential}

The macroscopic Gibbs potential (24) is that of a thermoelastic material. The corresponding macroscopic strain $\overline{\boldsymbol{\epsilon}}=(\partial G / \partial \overline{\boldsymbol{\sigma}})_{T}$ takes the form

$$
\overline{\boldsymbol{\epsilon}}=\boldsymbol{M}^{\mathrm{eff}} \overline{\boldsymbol{\sigma}}+\boldsymbol{m}^{\mathrm{eff}},
$$

where the effective compliance tensor, $\boldsymbol{M}^{\mathrm{eff}}$, and the total eigenstrain, $\boldsymbol{m}^{\mathrm{eff}}$, are given by

$$
\boldsymbol{M}^{\mathrm{eff}}=\left\langle\boldsymbol{B}^{\top} \boldsymbol{M} \boldsymbol{B}\right\rangle=\langle\boldsymbol{M B}\rangle=\left\langle\boldsymbol{B}^{\top} \boldsymbol{M}\right\rangle,
$$

and

$$
\boldsymbol{m}^{\mathrm{eff}}=\left\langle\boldsymbol{B}^{\boldsymbol{\top}}(\boldsymbol{M} \boldsymbol{b}+\boldsymbol{m})\right\rangle=\langle\boldsymbol{M} \boldsymbol{b}+\boldsymbol{m}\rangle=\left\langle\boldsymbol{B}^{\boldsymbol{\top}} \boldsymbol{m}\right\rangle .
$$

From equation (28) and definition (2-1) of the CTE tensor, the effective CTE tensor $\boldsymbol{\alpha}^{\text {eff }}(\overline{\boldsymbol{\sigma}}, T)=(\partial \overline{\boldsymbol{\epsilon}} / \partial T)_{\overline{\boldsymbol{\sigma}}}$ is found to be given by

$$
\boldsymbol{\alpha}^{\mathrm{eff}}(\overline{\boldsymbol{\sigma}}, T)=\frac{d \boldsymbol{M}^{\mathrm{eff}}}{d T} \overline{\boldsymbol{\sigma}}+\frac{d\left\langle\boldsymbol{B}^{\boldsymbol{\top}} \boldsymbol{m}\right\rangle}{d T} .
$$

The effective CFTE tensor,

$$
\boldsymbol{\alpha}^{\mathrm{eff}}(\mathbf{0}, T)=\frac{d\left\langle\boldsymbol{B}^{\top} \boldsymbol{m}\right\rangle}{d T},
$$

can be additively decomposed as

$$
\begin{aligned}
\boldsymbol{\alpha}^{\mathrm{eff}}(\mathbf{0}, T)= & \frac{d}{d T}\left\langle\boldsymbol{B}^{\boldsymbol{\top}} \int_{T_{0}}^{T} \boldsymbol{\alpha}(\mathbf{0}, T)\right\rangle+\left\langle\frac{d \boldsymbol{B}^{\top}}{d T} \boldsymbol{m}_{*}\right\rangle \\
= & \left\langle\boldsymbol{B}^{\top} \boldsymbol{\alpha}(\mathbf{0}, T)\right\rangle+\left\langle\frac{d \boldsymbol{B}^{\top}}{d T} \boldsymbol{m}_{\mathrm{th}}\right\rangle \\
& +\left\langle\frac{d \boldsymbol{B}^{\top}}{d T} \boldsymbol{m}_{*}\right\rangle .
\end{aligned}
$$

The first term on the right-hand side represents the estimate one obtains if one neglects the temperature dependence of the elasticity tensors of the constituents, whether a superimposed eigenstrain is present or not. The second term is a correction term accounting for the temperature dependence of the elasticity tensors of the constituents in the absence of any superimposed eigenstrain. The third term captures the effects of the superimposed eigenstrain. From the form of this term, it follows that if the elasticity tensors of the constituents depend on temperature, then (i) the superimposed eigenstrain enters the expression of the 
effective CFTE tensor and (ii) the effective CFTE tensor depends on temperature, even if the CFTE tensors of the constituents are temperature independent. This confirms that a framework broader than linear thermoelasticity, in which the elasticity tensors are assumed temperature-independent, is needed to capture the effects of the superimposed eigenstrain.

The macroscopic entropy $\bar{\eta}(\overline{\boldsymbol{\sigma}}, T)=-(\partial G / \partial T)_{\overline{\boldsymbol{\sigma}}}$ may be written as

$$
\begin{aligned}
\bar{\eta}= & \frac{1}{2} \overline{\boldsymbol{\sigma}} \cdot \frac{d \boldsymbol{M}^{\mathrm{eff}}}{d T} \overline{\boldsymbol{\sigma}}+\frac{d \boldsymbol{m}^{\mathrm{eff}}}{d T} \cdot \overline{\boldsymbol{\sigma}}+\frac{1}{2} \frac{d}{d T}\langle\boldsymbol{b} \cdot \boldsymbol{m}\rangle \\
& +\left\langle\int_{T_{0}}^{T} \frac{C_{\boldsymbol{\sigma}}(\mathbf{0}, \nu)}{\nu} d \nu\right\rangle+\left\langle\eta_{0}\right\rangle,
\end{aligned}
$$

so that the effective heat capacity at constant stress $C_{\boldsymbol{\sigma}}^{\text {eff }}(\overline{\boldsymbol{\sigma}}, T)=T(\partial \bar{\eta} / \partial T)_{\overline{\boldsymbol{\sigma}}}$ is given by

$$
\begin{aligned}
C_{\boldsymbol{\sigma}}^{\mathrm{eff}}(\overline{\boldsymbol{\sigma}}, T)= & \left\langle C_{\boldsymbol{\sigma}}(\mathbf{0}, T)\right\rangle+\frac{1}{2} T \frac{d^{2}}{d T^{2}}\langle\boldsymbol{b} \cdot \boldsymbol{m}\rangle \\
& +T\left(\frac{1}{2} \overline{\boldsymbol{\sigma}} \cdot \frac{d^{2} \boldsymbol{M}^{\mathrm{eff}}}{d T^{2}} \overline{\boldsymbol{\sigma}}+\frac{d^{2} \boldsymbol{m}^{\mathrm{eff}}}{d T^{2}} \cdot \overline{\boldsymbol{\sigma}}\right)
\end{aligned}
$$

For a reference stress $\boldsymbol{\sigma}_{0}(\boldsymbol{x})$, the same reasoning leads to

$$
\begin{aligned}
C_{\boldsymbol{\sigma}}^{\mathrm{eff}}(\overline{\boldsymbol{\sigma}}, T)= & \left\langle C_{\boldsymbol{\sigma}}\left(\boldsymbol{\sigma}_{0}, T\right)\right\rangle+\frac{1}{2} T \frac{d^{2}}{d T^{2}}\langle\boldsymbol{b} \cdot \boldsymbol{m}\rangle \\
& -T\left(\frac{1}{2}\left\langle\boldsymbol{\sigma}_{0} \cdot \frac{d^{2} \boldsymbol{M}}{d T^{2}} \boldsymbol{\sigma}_{0}\right\rangle+\left\langle\boldsymbol{\sigma}_{0} \cdot \frac{d^{2} \boldsymbol{m}}{d T^{2}}\right\rangle\right) \\
& +T\left(\frac{1}{2} \overline{\boldsymbol{\sigma}} \cdot \frac{d^{2} \boldsymbol{M}^{\mathrm{eff}}}{d T^{2}} \overline{\boldsymbol{\sigma}}+\frac{d^{2} \boldsymbol{m}^{\mathrm{eff}}}{d T^{2}} \cdot \overline{\boldsymbol{\sigma}}\right) .
\end{aligned}
$$

Within linear thermoelasticity, the elasticity tensors and CFTEs of the constituents are temperature independent, so that $\boldsymbol{B}$ is temperature independent and $\boldsymbol{m}, \boldsymbol{b}$, and $\boldsymbol{m}^{\text {eff }}$ are affine in $T$. This and equation (35) (or (36)) imply that the superimposed eigenstrain has no effect on $C_{\boldsymbol{\sigma}}^{\text {eff }}(\overline{\boldsymbol{\sigma}}, T)$ within that framework.

The conclusions below follow from expressions (29), (30), (32), (33), (35), and (36) for the overall moduli. A sentence of the form "the superimposed eigenstrain affects some overall modulus" means that the superimposed eigenstrain occurs in the expression for that modulus, but nothing is implied about the significance of the effects of the superimposed eigenstrain on the modulus.

- The elasticity tensors of the constituents being assumed to be independent of the superimposed eigenstrain, the stress concentration tensor $\boldsymbol{B}$ is unaffected by the superimposed eigenstrain, and so are the effective elasticity tensor.

- The superimposed eigenstrain affects the effective total eigenstrain.

- The superimposed eigenstrain, in general, affects the effective CFTE tensor. Its effect is made possible by the temperature dependence of the elasticity tensors of the constituents. If the elasticity tensors of the constituents are temperature independent, then the superimposed eigenstrain has no effect on the effective CFTE tensor even if the CFTE tensors of the constituents depend on temperature. 
- The superimposed eigenstrain, in general, induces anisotropy in both the free thermal expansion response and CFTE tensor in an otherwise isotropic composite (see the third term on the right-hand-side of (33)).

- In the limiting case of equal constituent CFTE tensors, the presence of a superimposed eigenstrain makes it possible for the effective CFTE tensor to differ from the common value of the constituent CFTE tensors. In particular, the composite can be thermally deformable even when the constituents are thermally undeformable, provided that the elasticity tensors of the constituents are temperature dependent. By (33), the effective thermal expansion of a composite with thermally undeformable constituents is $\left\langle\left(d \boldsymbol{B}^{\top} / d T\right) \boldsymbol{m}_{*}\right\rangle$.

- The superimposed eigenstrain, in general, affects the effective heat capacity at constant stress, $C_{\boldsymbol{\sigma}}$. Unlike in the case of the effective CFTEs, the temperature independence of the elasticity tensors of the constituents is not a sufficient condition for the superimposed eigenstrain to disappear from the expression of $C_{\boldsymbol{\sigma}}$. Assume that the elasticity tensors of the constituents do not depend on temperature but that their CFTEs do. Then, $\boldsymbol{b}$ is, in general, not affine in $T$, so that the term $d^{2}\left\langle\boldsymbol{b} \cdot \boldsymbol{m}_{*}\right\rangle / d T^{2}$ in (35) or (36) does not vanish. For the same reason, the superimposed eigenstrain does not, in general, disappear from the expression of $C_{\boldsymbol{\sigma}}$ when the elasticity tensors of the constituents depend on temperature, even if the CFTE tensors of the constituents do not depend on temperature.

- The superimposed eigenstrain has no effect on the effective moduli if it is uniform.

\subsection{Values deduced from the macroscopic Helmholtz po- tential}

Following the same steps as for the macroscopic Gibbs potential, one can show that the effective stiffness tensor, $\boldsymbol{L}^{\mathrm{eff}}$, the effective eigenstress, $\boldsymbol{l}^{\text {eff }}$, and the effective stress-temperature tensor, $\boldsymbol{\beta}^{\text {eff }}(\overline{\boldsymbol{\sigma}}, T)$, are given by

$$
\begin{gathered}
\boldsymbol{L}^{\mathrm{eff}}=\left\langle\boldsymbol{A}^{\top} \boldsymbol{L} \boldsymbol{A}\right\rangle=\langle\boldsymbol{L} \boldsymbol{A}\rangle=\left\langle\boldsymbol{A}^{\top} \boldsymbol{L}\right\rangle, \\
\boldsymbol{l}^{\mathrm{eff}}=\left\langle\boldsymbol{A}^{\top}(\boldsymbol{L} \boldsymbol{a}+\boldsymbol{l})\right\rangle=\left\langle\boldsymbol{A}^{\top} \boldsymbol{l}\right\rangle=\langle\boldsymbol{L} \boldsymbol{a}+\boldsymbol{l}\rangle, \\
\boldsymbol{\beta}^{\mathrm{eff}}(\overline{\boldsymbol{\epsilon}}, T)=\frac{d \boldsymbol{L}^{\mathrm{eff}}}{d T} \overline{\boldsymbol{\epsilon}}+\frac{d\left\langle\boldsymbol{A}^{\top} \boldsymbol{l}\right\rangle}{d T},
\end{gathered}
$$

and that the effective heat capacity at constant strain $C_{\boldsymbol{\epsilon}}^{\text {eff }}(\overline{\boldsymbol{\epsilon}}, T)$ is given by either

$$
\begin{aligned}
C_{\boldsymbol{\epsilon}}^{\mathrm{eff}}(\overline{\boldsymbol{\epsilon}}, T)= & \left\langle C_{\boldsymbol{\epsilon}}(\mathbf{0}, T)\right\rangle-\frac{1}{2} T \frac{d^{2}}{d T^{2}}\langle\boldsymbol{a} \cdot \boldsymbol{l}\rangle \\
& -T\left(\overline{\boldsymbol{\epsilon}} \cdot \frac{d^{2} \boldsymbol{L}^{\mathrm{eff}}}{d T^{2}} \overline{\boldsymbol{\epsilon}}+\frac{d^{2} \boldsymbol{l}^{\mathrm{eff}}}{d T^{2}} \cdot \overline{\boldsymbol{\epsilon}}\right),
\end{aligned}
$$


or

$$
\begin{aligned}
C_{\boldsymbol{\epsilon}}^{\mathrm{eff}}(\overline{\boldsymbol{\epsilon}}, T)= & \left\langle C_{\boldsymbol{\epsilon}}\left(\boldsymbol{\epsilon}_{0}, T\right)\right\rangle-\frac{1}{2} T \frac{d^{2}}{d T^{2}}\langle\boldsymbol{a} \cdot \boldsymbol{l}\rangle \\
& +T\left(\frac{1}{2}\left\langle\boldsymbol{\epsilon}_{0} \cdot \frac{d^{2} \boldsymbol{L}}{d T^{2}} \boldsymbol{\epsilon}_{0}\right\rangle+\left\langle\boldsymbol{\epsilon}_{0} \cdot \frac{d^{2} \boldsymbol{l}}{d T^{2}}\right\rangle\right) \\
& -T\left(\overline{\boldsymbol{\epsilon}} \cdot \frac{d^{2} \boldsymbol{L}^{\mathrm{eff}}}{d T^{2}} \overline{\boldsymbol{\epsilon}}+\frac{d^{2} \boldsymbol{l}^{\mathrm{eff}}}{d T^{2}} \cdot \overline{\boldsymbol{\epsilon}}\right)
\end{aligned}
$$

depending on the choice of the reference strain.

\section{Case of the two-phase composite}

This section considers two-phase composites, for which Levin-type formulas are given. It is assumed that the superimposed eigenstrain is uniform over each phase. Let $\boldsymbol{m}_{*}^{(1)}$ and $\boldsymbol{m}_{*}^{(2)}$ denote the values of the superimposed eigenstrains in phases 1 and 2 , respectively.

If the stress and temperature are used as independent variables, the following Levin-type formulas can be obtained by using the usual arguments:

$$
\begin{aligned}
\boldsymbol{m}^{\mathrm{eff}}= & \langle\boldsymbol{m}\rangle+\left(\boldsymbol{M}^{\mathrm{eff}}-\langle\boldsymbol{M}\rangle\right)[\boldsymbol{M}]^{-1}[\boldsymbol{m}], \\
\boldsymbol{\alpha}^{\mathrm{eff}}(\mathbf{0}, T)= & \langle\boldsymbol{\alpha}(\mathbf{0}, T)\rangle \\
& +\frac{d}{d T}\left(\left(\boldsymbol{M}^{\mathrm{eff}}-\langle\boldsymbol{M}\rangle\right)[\boldsymbol{M}]^{-1}[\boldsymbol{m}]\right), \\
C_{\boldsymbol{\sigma}}^{\mathrm{eff}}(\mathbf{0}, T)= & \left\langle C_{\boldsymbol{\sigma}}(\mathbf{0}, T)\right\rangle \\
& +\frac{1}{2} T \frac{d^{2}}{d T^{2}}\left([\boldsymbol{M}]^{-1}[\boldsymbol{m}]\right. \\
& \left.\cdot\left(\boldsymbol{M}^{\mathrm{eff}}-\langle\boldsymbol{M}\rangle\right)[\boldsymbol{M}]^{-1}[\boldsymbol{m}]\right),
\end{aligned}
$$

where

$$
[\boldsymbol{m}]=\int_{T_{0}}^{T}[\boldsymbol{\alpha}(\mathbf{0}, T)] d t+\left[\boldsymbol{m}_{*}\right]
$$

The square brackets denote the difference in the bracketed quantity between phase 1 and phase 2, e.g., $[\boldsymbol{M}]=\boldsymbol{M}^{(1)}-\boldsymbol{M}^{(2)},[\boldsymbol{m}]=\boldsymbol{m}^{(1)}-\boldsymbol{m}^{(2)}$. These formulas show that $\boldsymbol{\alpha}^{\mathrm{eff}}(\mathbf{0}, T)$ and $C_{\boldsymbol{\sigma}}^{\mathrm{eff}}(\mathbf{0}, T)$ depend on the superimposed eigenstrains $\boldsymbol{m}_{*}^{(1)}$ and $\boldsymbol{m}_{*}^{(2)}$ only through their difference.

If the strain and temperature are used as independent variables, formulas analogous to the above can be obtained by using the same type of arguments. E.g.,

$$
\begin{aligned}
\boldsymbol{l}^{\mathrm{eff}}= & \langle\boldsymbol{l}\rangle+\left(\boldsymbol{L}^{\mathrm{eff}}-\langle\boldsymbol{L}\rangle\right)[\boldsymbol{L}]^{-1}[\boldsymbol{l}], \\
\boldsymbol{\beta}^{\mathrm{eff}}(\mathbf{0}, T)= & \langle\boldsymbol{\beta}(\mathbf{0}, T)\rangle \\
& +\frac{d}{d T}\left(\left(\boldsymbol{L}^{\mathrm{eff}}-\langle\boldsymbol{L}\rangle\right)[\boldsymbol{L}]^{-1}[\boldsymbol{l}]\right), \\
C_{\boldsymbol{\epsilon}}^{\mathrm{eff}}(\mathbf{0}, T)= & \left\langle C_{\boldsymbol{\epsilon}}(\mathbf{0}, T)\right\rangle
\end{aligned}
$$




$$
\begin{aligned}
-\frac{1}{2} T \frac{d^{2}}{d T^{2}}\left([\boldsymbol{L}]^{-1}[\boldsymbol{l}]\right. \\
\left.\cdot\left(\boldsymbol{L}^{\mathrm{eff}}-\langle\boldsymbol{L}\rangle\right)[\boldsymbol{L}]^{-1}[\boldsymbol{l}]\right) .
\end{aligned}
$$

\section{Numerical example: Titanium alloy matrix with zirconium oxide inclusions}

This section discusses a numerical example related to high temperature applications. The example data are drawn from the literature on functionally graded materials (Tanigawa et al. (1997); Ootao et al. (1998, 1999a,b); Wang and Mai (2005); Ching and Chen (2007)). For the proper modeling of these materials at high-temperatures, consideration of the effects of the local residual stresses and those of the material temperature dependence is regarded as critical (Birman and Byrd (2007)).

Below, the magnitude of a (symmetric) tensor quantity will refer to the absolute value of its eigenvalue with largest absolute value, and the order of magnitude of a (symmetric) tensor quantity will refer to the order of magnitude of its eigenvalue with largest absolute value.

\subsection{Example data}

\subsubsection{Temperature range}

The effective moduli are estimated over the temperature range of 300 to $1300 \mathrm{~K}$. It is the same as that used by Tanigawa et al. (1997) in some of their analyses involving the composite considered here.

\subsubsection{Constituents}

The composite material considered is two-phase, consisting of $\mathrm{ZrO}_{2}$ inclusions embedded in a $\mathrm{Ti}-6 \mathrm{Al}-4 \mathrm{~V}$ matrix. Both constituents are assumed to be isotropic. The materials' moduli are represented over the temperature range by the best fit expressions given in Table 1.

The coefficient of free linear thermal expansion of $\mathrm{Ti}-6 \mathrm{Al}-4 \mathrm{~V}$, which is defined piecewise, increases with temperature and plateaus above around $1100 \mathrm{~K}$. As interpolated above, it is not $T$-differentiable at $T=1100 \mathrm{~K}$. In the results below, the plateau is made to begin at the temperature at which the coefficient reaches its maximum, that is, at $1033.46 \mathrm{~K}$ (instead of $1100 \mathrm{~K}$ ) and its value is fixed at $10.303 \times 10^{-6} \mathrm{~K}^{-1}$ (instead of $10.291 \times 10^{-6} \mathrm{~K}^{-1}$ ). This slight modification, which negligibly affects the predicted effective moduli, removes the artificial discontinuity introduced by the interpolation.

Figures 1-4 sum up the data for the constituents. In these figures, $G, K$, $\alpha$, and $C_{p}$ generically denote shear modulus, bulk modulus, coefficient of free linear thermal expansion, and heat capacity per unit reference volume at constant pressure, respectively. For $\mathrm{ZrO}_{2}$, the shear and bulk moduli are both two (at higher temperatures) to three (at lower temperatures) orders of magnitude larger (in absolute value) than their respective $T$-derivatives, which are in turn three orders of magnitude larger than their respective $T$-derivatives. For $\mathrm{Ti}-$ $6 \mathrm{Al}-4 \mathrm{~V}$, the shear and bulk moduli and the $T$-derivative of the bulk modulus 
are each three orders of magnitude larger than their respective $T$-derivatives, whereas the $T$-derivative of the shear modulus is four orders of magnitude larger than its $T$-derivative.

\subsubsection{Aspect ratio and composition}

The $\mathrm{ZrO}_{2}$ inclusions are assumed to be spherical and randomly distributed within the matrix, so that the overall behavior of the composite is isotropic in the absence of superimposed eigenstrain. Their volume fraction is fixed at $20 \%$.

\subsubsection{Superimposed eigenstrain field}

The superimposed eigenstrain field used in the simulations, assumed to be phasewise uniform, is such that

$$
\boldsymbol{m}_{*}^{(\mathrm{m})}-\boldsymbol{m}_{*}^{(\mathrm{i})}=10^{-3}\left(\boldsymbol{e}_{1} \otimes \boldsymbol{e}_{1}+2 \boldsymbol{e}_{2} \otimes \boldsymbol{e}_{2}\right),
$$

where superscripts $\mathrm{m}$ and i refer to the matrix phase and the inclusion phase, respectively.

\subsubsection{Reference temperature for thermal expansions and reference pressure for heat capacities}

The reference temperature for zero thermal eigenstrain is taken as $T_{\text {ref }}=300 \mathrm{~K}$. The CTEs given above are assumed to be the CFTEs of the constituents. In view of the orders of magnitude of the eigenvalues of the compliance tensor $T$-derivatives discussed below, one can check that the CFTEs and the CTEs of the constituents, which are related by (12), are very close at the atmospheric pressure ( $\boldsymbol{\sigma}_{0} \approx 10^{5} \boldsymbol{i} \mathrm{Pa}, \boldsymbol{i}$ being the second-order identity tensor).

The constituent heat capacities given above are assumed to be the constituent heat capacities at atmospheric pressure. Below, the effective heat capacity $C_{\boldsymbol{\sigma}}^{\text {eff }}$ is also estimated at $\boldsymbol{\sigma}=\boldsymbol{\sigma}_{0}$,

$$
\begin{aligned}
C_{\boldsymbol{\sigma}}^{\mathrm{eff}}\left(\boldsymbol{\sigma}_{0}, T\right)= & \left\langle C_{\boldsymbol{\sigma}}\left(\boldsymbol{\sigma}_{0}, T\right)\right\rangle+\frac{1}{2} T \frac{d^{2}}{d T^{2}}\langle\boldsymbol{b} \cdot \boldsymbol{m}\rangle \\
& +\frac{T}{2} \boldsymbol{\sigma}_{0} \cdot\left(\frac{d^{2} \boldsymbol{m}^{\mathrm{eff}}}{d T^{2}}-\left\langle\frac{d^{2} \boldsymbol{m}}{d T^{2}}\right\rangle\right) \\
& +\frac{T}{2} \boldsymbol{\sigma}_{0} \cdot\left(\frac{d^{2} \boldsymbol{M}^{\mathrm{eff}}}{d T^{2}}-\left\langle\frac{d^{2} \boldsymbol{M}}{d T^{2}}\right\rangle\right) \boldsymbol{\sigma}_{0} .
\end{aligned}
$$

\subsection{Effective moduli}

\subsubsection{Micromechanical model}

The Mori-Tanka model is used to estimate the effective moduli of the composite. In the case of two-phase inclusion-matrix composites, the equations defining the model include the following:

$$
\boldsymbol{B}^{(\mathrm{m})}=\left(c^{(\mathrm{m})} \boldsymbol{I}+c^{(\mathrm{i})} \boldsymbol{L}^{(\mathrm{i})}(\boldsymbol{I}-\boldsymbol{P}[\boldsymbol{L}])^{-1} \boldsymbol{M}^{(\mathrm{m})}\right)^{-1},
$$




$$
\boldsymbol{b}^{(\mathrm{m})}=\left(\boldsymbol{B}^{(\mathrm{m})}-\boldsymbol{I}\right)[\boldsymbol{M}]^{-1}[\boldsymbol{m}],
$$

where $\boldsymbol{B}^{(\mathrm{m})}$ and $\boldsymbol{b}^{(\mathrm{m})}$ denote the Mori-Tanaka estimates to the averages over the matrix phase of $\boldsymbol{B}$ and $\boldsymbol{b}$, respectively; $c^{(\mathrm{m})}$ and $c^{(\mathrm{i})}$ are the volume fractions of the matrix and the inclusions, respectively; $\boldsymbol{I}$ is the symmetric fourth-order identity tensor; $[\boldsymbol{L}]=\boldsymbol{L}^{(m)}-\boldsymbol{L}^{(i)} ;[\boldsymbol{M}]=\boldsymbol{M}^{(m)}-\boldsymbol{M}^{(i)} ;[\boldsymbol{m}]=\boldsymbol{m}^{(m)}-\boldsymbol{m}^{(i)}$; and $\boldsymbol{P}$ is the Hill polarization tensor.

Computing the effective moduli requires the first and second order $T$-derivatives of the above and similar quantities. Numerical differentiation is one option, which was discussed by Dvorak (2012, p. 252). Analytical differentiation offers no difficulty in principle. In practice, however, the computation of the first and second $T$-derivatives of $\boldsymbol{P}$ is analytically impractical in the general case. A sense of the difficulty can be gained by consulting (Suvorov and Dvorak, 2002) where analytical expressions were derived for the first-order T-derivative of $\boldsymbol{P}$ in the cases of inclusions with aspect ratios of 0,1 and infinity. For this study, a Mathematica package was developed that estimates the effective moduli for arbitrary shape-factor inclusions in an isotropic matrix. For this, techniques akin to automatic differentiation were implemented by using Mathematica's command OptimizeExpression in the Experimental package.

\subsubsection{Effective elasticities}

The eigenvalues and their first and second $T$-derivatives as functions of temperature for stress concentration factor $\boldsymbol{B}^{(\mathrm{m})}$ and effective compliance tensor $\boldsymbol{M}^{\text {eff }}$ are given in figures referred to in the discussion to follow.

\subsubsection{Effective thermal expansion}

The first (respectively second, third) term on the right-hand side of decomposition (33) of the effective CFTE tensor will subsequently be referred to as Term 1 (respectively 2,3).

For the composite under consideration, Terms 1 and 2 are isotropic, because tensors $\boldsymbol{B}, \boldsymbol{m}_{\mathrm{th}}$ and their $T$-derivatives are isotropic. Term 3 , in contrast, is anisotropic.

As expected, the higher the temperature, the more apparent the combined effects of the material temperature dependence and the presence of the superimposed eigenstrains. Thus, below $760 \mathrm{~K}$, the magnitudes of Terms 2 and 3 are both less than $1 \%$ of that of Term 1, while, at $1190 \mathrm{~K}$, both magnitudes exceed $5 \%$ of that of Term 1 . At $1300 \mathrm{~K}$, these magnitudes are respectively larger than $14 \%$ and $11 \%$ of the magnitude of Term 1 . The sum of Terms 2 and 3 accounts for $20 \%$ of component 22 of the effective CFTE tensor with superimposed eigenstrain (Figure 5). Also, the anisotropy induced by the superimposed eigenstrain increases with increasing temperature: It is imperceptible for small to moderate temperature changes, becoming evident in the upper part of the temperature range (Figure 6).

Term 3 dominates Term 2 for a good portion of the temperature range. The magnitude of Term 2, which is zero at the lower end of the temperature range and increases with increasing temperature, reaches the value of the smallest eigenvalue of Term 3 at around $500 \mathrm{~K}$, that of the intermediate eigenvalue at 
around $700 \mathrm{~K}$, and that of the largest eigenvalue at about $1100 \mathrm{~K}$. Above $1100 \mathrm{~K}$, the magnitude of Term 2 exceeds that of Term 3 (Figure 7).

The sharp increase of Terms 2 and 3 starting around $900 \mathrm{~K}$ can be traced to a sharp increase in the magnitude of $d \boldsymbol{B}^{(\mathrm{m}) T} / d T$ around that temperature (Figure 8-b). The sharp increase of $d \boldsymbol{B}^{(\mathrm{m}) T} / d T$ can in turn be traced to the relative loss of stiffness of the inclusions with increasing temperature (Figures 1,2 and 8). To illustrate, the magnitude of $d \boldsymbol{M}_{\mathrm{ZrO}_{2}} / d T$ increases by more than two orders of magnitude over the temperature range (Figure 9-a), whereas the magnitude of $d \boldsymbol{M}_{\mathrm{Ti}-6 \mathrm{Al}-4 \mathrm{~V}} / d T$ increases by less than one order of magnitude (Figure 9-b).

\subsubsection{Effective heat capacity at constant pressure per unit reference volume}

The effective heat capacities predicted by the present approach are very close to those predicted by the law of mixtures. The heat capacities are thus negligibly affected by the consideration of all the correction terms introduced by the thermoelastic coupling, let alone by the superimposed eigenstrains. This can be checked by examining the orders of magnitude of the various terms occurring in expression (38) for $C_{\boldsymbol{\sigma}}$ :

- The order-of-magnitude of term $\left\langle C_{\boldsymbol{\sigma}}\left(\boldsymbol{\sigma}_{0}, T\right)\right\rangle$ is $10^{6} \mathrm{~J} \cdot \mathrm{m}^{-3} \cdot \mathrm{K}^{-1}$ (Figure 4).

- The order-of-magnitude of term $(T / 2) d^{2}\langle\boldsymbol{b} \cdot \boldsymbol{m}\rangle / d T^{2}$ is $10^{3} \mathrm{~J} \cdot \mathrm{m}^{-3} \cdot \mathrm{K}^{-1}$.

- The order-of-magnitude of term $(T / 2) \boldsymbol{\sigma}_{0} \cdot\left(d^{2} \boldsymbol{m}^{\text {eff }} / d T^{2}-\left\langle d^{2} \boldsymbol{m} / d T^{2}\right\rangle\right)$ is $1 \mathrm{~J} \cdot \mathrm{m}^{-3} \cdot \mathrm{K}^{-1}$.

- The order-of-magnitude of term (T/2) $\boldsymbol{\sigma}_{0} \cdot\left(d^{2} \boldsymbol{M}^{\mathrm{eff}} / d T^{2}-\left\langle d^{2} \boldsymbol{M} / d T^{2}\right\rangle\right) \boldsymbol{\sigma}_{0}$ is $10^{-2} \mathrm{~J} \cdot \mathrm{m}^{-3} \cdot \mathrm{K}^{-1}$.

These orders of magnitude can be obtained by noting that the orders of magnitude of the individual quantities entering these terms are as follows. The order of magnitude of $\boldsymbol{b}$ is $10^{8} \mathrm{~Pa}$, that of $d \boldsymbol{b} / d T$ is $10^{5} \mathrm{~Pa} \cdot \mathrm{K}^{-1}$, and that of $d^{2} \boldsymbol{b} / d T^{2}$ is $10^{3} \mathrm{~Pa} \cdot \mathrm{K}^{-2}$ (Figure 10). The maximum order of magnitude of terms $\boldsymbol{m}$ is $10^{-2}$, that of terms $d \boldsymbol{m} / d T$ is $10^{-5} \mathrm{~K}^{-1}$, and that of terms $d^{2} \boldsymbol{m} / d T^{2}$ is $10^{-8} \mathrm{~K}^{-2}$ (Figures 3 and 6). The maximum order of magnitude of $T$ is $10^{3} \mathrm{~K}$. The order of magnitude of the second $T$-derivatives of the compliances varies markedly with temperature. The order of magnitude of the second $T$-derivative of $M_{\mathrm{ZrO}_{2}}$ increases from $10^{-17} \mathrm{~Pa}^{-1} \cdot \mathrm{K}^{-2}$ at $300 \mathrm{~K}$ to $10^{-14} \mathrm{~Pa}^{-1} \cdot \mathrm{K}^{-2}$ at $1300 \mathrm{~K}$ (Figure 9 -a); that of $\boldsymbol{M}_{\mathrm{Ti}-6 \mathrm{Al}-4 \mathrm{~V}}$, from $10^{-17} \mathrm{~Pa}^{-1} \cdot \mathrm{K}^{-2}$ at $300 \mathrm{~K}$ to $10^{-16} \mathrm{~Pa}^{-1} \cdot \mathrm{K}^{-2}$ at $1300 \mathrm{~K}$ (Figure 9-b); that of $\boldsymbol{M}^{\mathrm{eff}}$, from $10^{-17} \mathrm{~Pa}^{-1} \cdot \mathrm{K}^{-2}$ at $300 \mathrm{~K}$ to $10^{-15} \mathrm{~Pa}^{-1} \cdot \mathrm{K}^{-2}$ at $1300 \mathrm{~K}$ (Figure $9-\mathrm{c}$ ).

The conclusion that the effective heat capacities of the composite predicted by the present approach and those predicted by the law of mixtures are similar is expected to hold true for other structural materials at room and higher temperatures, at which the heat capacity per unit volume has an order of magnitude of $10^{6} \mathrm{~J} \cdot \mathrm{m}^{-3} \cdot \mathrm{K}^{-1}$ (Hatta, 2006). Such a conclusion, however, may not hold in the case of low temperatures, in which the orders of magnitude of the heat capacities are much lower. 


\section{Conclusions}

The effects that the presence of a eigenstrain field, when superimposed on the thermal eigenstrain, has on the overall thermoelastic moduli of composites were quantified within the framework of small strain thermoelasticity with temperature dependent materials. Expressions for the Gibbs and Helmholtz potentials of a homogeneous thermoelastic material with a superimposed eigenstrain were first developed. Combining these potentials with the concentration relations then yielded the Gibbs and Helmholtz potentials of the heterogeneous thermoelastic material with a superimposed eigenstrain field, from which expressions for the effective CTE tensor, stress-temperature tensor, and heat capacities were finally obtained. A numerical example was thoroughly investigated. Besides the effective moduli, the orders of magnitude of the various correction terms induced by the temperature dependence of the constituent moduli and the presence of the superimposed eigenstrain were discussed.

The paper provides a framework for modeling thermoelastic composites undergoing small thermoelastic strains and subjected to large temperature changes and possibly to superimposed eigenstrains.

\section{References}

Y. A. Bahei-El-Din and G. J. Dvorak. Isothermal fatigue behavior of Sigma/TIMETAL 21S laminates, Part II: Modeling and numerical analysis. Mechanics of Composite Materials and Structures, 4(2):131-158, 1997.

Y. Benveniste and G. J. Dvorak. On a correspondence between mechanical and thermal effects in two-phase composites. In G. J. Weng, M. Taya, and H. Abé, editors, Micromechanics and inhomogeneity: The Toshio Mura 65th anniversary volume, pages 65-81. Springer-Verlag, New York, NY, 1990.

V. Birman and L. W. Byrd. Modeling and analysis of functionally graded materials and structures. Applied Mechanics Reviews, 60(5):195-216, 2007.

F. Bobaru. Designing optimal volume fractions for functionally graded materials with temperature-dependent material properties. Journal of Applied Mechanics-Transactions of the ASME, 74:861-874, 2007.

D. Boussaa. Optimization of temperature-dependent functionally graded material bodies. Computer Methods in Applied Mechanics and Engineering, 198 (37-40):2827-2838, 2009.

D. Boussaa. Effective thermoelastic properties of composites with temperaturedependent constituents. Mechanics of Materials, 43(8):397-407, 2011.

Tianbao Cheng, Weiguo Li, Yushan Shi, Wei Lu, and Daining Fang. Effects of mechanical boundary conditions on thermal shock resistance of ultra-high temperature ceramics. Applied Mathematics and Mechanics, 36(2):201-210, 2015.

H. K. Ching and J. K. Chen. Thermal stress analysis of functionally graded composites with temperature-dependent material properties. Journal of $\mathrm{Me}$ chanics of Materials and Structures, 2(4):633-653, 2007. 
D. de Faoite, D. J. Browne, and K. T. Stanton. Regression analysis of temperature-dependent mechanical and thermal properties of dielectric technical ceramics. Journal of Materials Science, 48(1):451-461, 2013.

G. J. Dvorak. Micromechanics of composite materials, volume 186 of Solid mechanics and its applications. Springer, New York, 2012.

T. R. Finlayson, E. E. Gibbs, and T. F. Smith. The low-temperature thermal expansion of polycrystalline $\mathrm{V}_{3}$ Ge. Physica $B+C, 108(1-3): 1011-1012,1981$.

E. E. Gibbs, T. R. Finlayson, and T. F. Smith. Anisotropic thermal expansion of polycrystalline V25.75at\%Si. Solid State Communications, 37(1):33-35, 1981.

Jie-Cai Han and Bao-Lin Wang. Thermal shock resistance of ceramics with temperature-dependent material properties at elevated temperature. Acta Materialia, 59(4):1373 - 1382, 2011.

I. Hatta. Heat capacity per unit volume. Thermochimica Acta, 446(1-2):176$179,2006$.

A.D. Kovalenko. Osnovy termouprugosti. Naukova Dumka, Kiev, 1970.

V. A. Lubarda. On thermodynamic potentials in linear thermoelasticity. International Journal of Solids and Structures, 41(26):7377-7398, 2004.

J. C. Nadeau and M. Ferrari. Effective thermal expansion of heterogeneous materials with application to low temperature environments. Mechanics of Materials, 36(3):201-214, 2004.

Y. Ootao, R. Kawamura, Y. Tanigawa, and T. Nakamura. Neural network optimization of material composition of a functionally graded material plate at arbitrary temperature range and temperature rise. Archive of Applied Mechanics, 68:662-676, 1998.

Y. Ootao, R. Kawamura, Y. Tanigawa, and R. Imamura. Optimization of material composition of nonhomogeneous hollow sphere for thermal stress relaxation making use of neural network. Computer Methods in Applied Mechanics and Engineering, 180:185-201, 1999a.

Y. Ootao, Y. Tanigawa, and T. Nakamura. Optimization of material composition of FGM hollow circular cylinder under thermal loading: A neural network approach. Composites Part B-Engineering, 30:415-422, $1999 \mathrm{~b}$.

A. P. Suvorov and G. J. Dvorak. Rate form of the Eshelby and Hill tensors. International Journal of Solids and Structures, 39(21-22):5659-5678, 2002.

Y. Tanigawa, M. Matsumoto, and T. Akai. Optimization of material composition to minimize thermal stresses in nonhomogeneous plate subjected to unsteady heat supply. JSME International Journal Series A, 40:84-93, 1997.

B. L. Wang and Y. W. Mai. Transient one-dimensional heat conduction problems solved by finite element. International Journal of Mechanical Sciences, 47:303-317, 2005. 
J. Wippler, S. Fünfschilling, F. Fritzen, T. Böhlke, and M. J. Hoffmann. Homogenization of the thermoelastic properties of silicon nitride. Acta Materialia, 59(15):6029-6038, 2011. 


\section{List of Figures}

1 Constituent shear moduli (a) and their respective first (b) and second (c) T-derivatives as functions of temperature. . . . . . . . 23

2 Constituent bulk moduli (a) and their respective first (b) and second (c) T-derivatives as functions of temperature. . . . . . . . 24

3 Constituent coefficients of free linear thermal expansion (b), their respective integrals from $T_{\text {ref }}$ (i.e., the constituent free linear thermal expansions from $T_{\text {ref }}$ ) (a) and their respective $T$-derivatives (c) as functions of temperature. . . . . . . . . . . . . . 25

4 Constituent heat capacities at constant pressure per unit reference volume as functions of temperature. . . . . . . . . . . . 26

5 Decomposition of the non-zero components of the effective CFTE tensor in the presence of a superimposed eigenstrain field $\boldsymbol{m}_{*}$ such that $\boldsymbol{m}_{*}^{(\mathrm{m})}-\boldsymbol{m}_{*}^{(\mathrm{i})}=10^{-3}\left(\boldsymbol{e}_{1} \otimes \boldsymbol{e}_{1}+2 \boldsymbol{e}_{2} \otimes \boldsymbol{e}_{2}\right)$. The curves labeled 1 correspond to Term 1 (i.e., $\left\langle\boldsymbol{B}^{\top} \boldsymbol{\alpha}\right\rangle$ ); the curves labeled 2 , to Term 2 (i.e., $\left.\left\langle(d \boldsymbol{B} / d T)^{\top} \boldsymbol{m}_{\mathrm{th}}\right\rangle\right)$; the curves labeled 3 , to Term 3 (i.e., $\left.\left\langle(d \boldsymbol{B} / d T)^{\top} \boldsymbol{m}_{*}\right\rangle\right)$. The gray curves correspond to the sum of the three terms, that is, to the non-zero components of $\boldsymbol{\alpha}^{\mathrm{eff}}(\mathbf{0}, T)$. . . . . . . . . . . . . . . . . .

6 Non-zero components (a) and their $T$-derivatives (b) as functions of temperature for the effective CFTE tensor in the presence of a superimposed eigenstrain field $\boldsymbol{m}_{*}$ such that $\boldsymbol{m}_{*}^{(\mathrm{m})}-$ $\boldsymbol{m}_{*}^{(\mathrm{i})}=10^{-3}\left(\boldsymbol{e}_{1} \otimes \boldsymbol{e}_{1}+2 \boldsymbol{e}_{2} \otimes \boldsymbol{e}_{2}\right)$. The curves labeled 1 (respectively 2,3$)$ represent component $\alpha_{11}^{\text {eff }}(\mathbf{0}, T)$ (a) and component $d \alpha_{11}^{\mathrm{eff}} / d T(\mathbf{0}, T)(\mathrm{b})$ (respectively $\alpha_{22}^{\mathrm{eff}}(\mathbf{0}, T)(\mathrm{a})$ and $d \alpha_{22}^{\mathrm{eff}} / d T(\mathbf{0}, T)$ (b), $\alpha_{33}^{\text {eff }}(\mathbf{0}, T)(\mathrm{a})$ and $\left.d \alpha_{33}^{\text {eff }} / d T(\mathbf{0}, T)(\mathrm{b})\right)$ of $\boldsymbol{\alpha}^{\text {eff }}(\mathbf{0}, T)$. The gray curves, which are hardly distinguishable from the curves labeled 3 , correspond to the value of the coefficient of free thermal expansion (a) and that of its $T$-derivative in the case of no superimposed eigenstrain. . . . . . . . . . . . . . . . . 28

7 Comparison of the non zero components of Term 2 (i.e., $\left.\left\langle(d \boldsymbol{B} / d T)^{\top} \boldsymbol{m}_{\mathrm{th}}\right\rangle\right)$ and Term 3 (i.e., $\left.\left\langle(d \boldsymbol{B} / d T)^{\top} \boldsymbol{m}_{*}\right\rangle\right)$ of the effective CFTE tensor in the presence of a superimposed eigenstrain field $\boldsymbol{m}_{*}$ such that $\boldsymbol{m}_{*}^{(\mathrm{m})}-\boldsymbol{m}_{*}^{(\mathrm{i})}=10^{-3}\left(\boldsymbol{e}_{1} \otimes \boldsymbol{e}_{1}+2 \boldsymbol{e}_{2} \otimes \boldsymbol{e}_{2}\right)$. The curve labeled 2 represents the diagonal term of Term 2 , which is isotropic. The curve labeled $3_{11}$ represents component 11 of Term 3 , and so on. The components of Term 2 are zero at $300 \mathrm{~K}$. Those of Term 3 are relatively small (around $10^{-8}$ ) but non-zero at $300 \mathrm{~K}$. . . . .

8 Eigenvalues of $\boldsymbol{B}^{(\mathrm{m})}$ (a) and their first (b) and second (c) $T$ derivatives as functions of temperature. The curves labeled 1 (respectively 2) correspond to the eigenvalue of multiplicity 5 (respectively 1). A linear scale is used for the ordinate axes of subfigures (a) and (b); a log scale, for the ordinate axes of subfigure $(c)$ and the inset in subfigure $(b) . \ldots . . . . . . .30$ 
9 Eigenvalues of the compliance tensors (curves labeled 1 and 2), those of their respective first (curves labeled $1^{\prime}$ and $2^{\prime}$ ) and second (curves labeled $1^{\prime \prime}$ and $2^{\prime \prime}$ ) $T$-derivatives as functions of temperature for $\mathrm{ZrO}_{2}$ (a), Ti6Al4V (b), and the composite (c). The curves labeled $1,1^{\prime}$, and $1^{\prime \prime}$ correspond to the eigenvalues of multiplicity $5 \ldots \ldots \ldots 31$

10 The three non vanishing components (a) and their first (b) and second (c) $T$-derivatives as functions of temperature for $\boldsymbol{b}^{(\mathrm{i})}$ in the presence of a superimposed eigenstrain field $\boldsymbol{m}_{*}$ such that $\boldsymbol{m}_{*}^{(\mathrm{m})}-\boldsymbol{m}_{*}^{(\mathrm{i})}=10^{-3}\left(\boldsymbol{e}_{1} \otimes \boldsymbol{e}_{1}+2 \boldsymbol{e}_{2} \otimes \boldsymbol{e}_{2}\right)$. The curves labeled 1 (respectively 2,3) represent components 11 (respectively 22, 33). The gray curves correspond to the case of no superimposed eigenstrain field, in which case $\boldsymbol{b}^{(\mathrm{i})}$ and its first and second $T$ derivatives are isotropic. In subfigure (c), the black curves are ordered as in subfigure (b). 

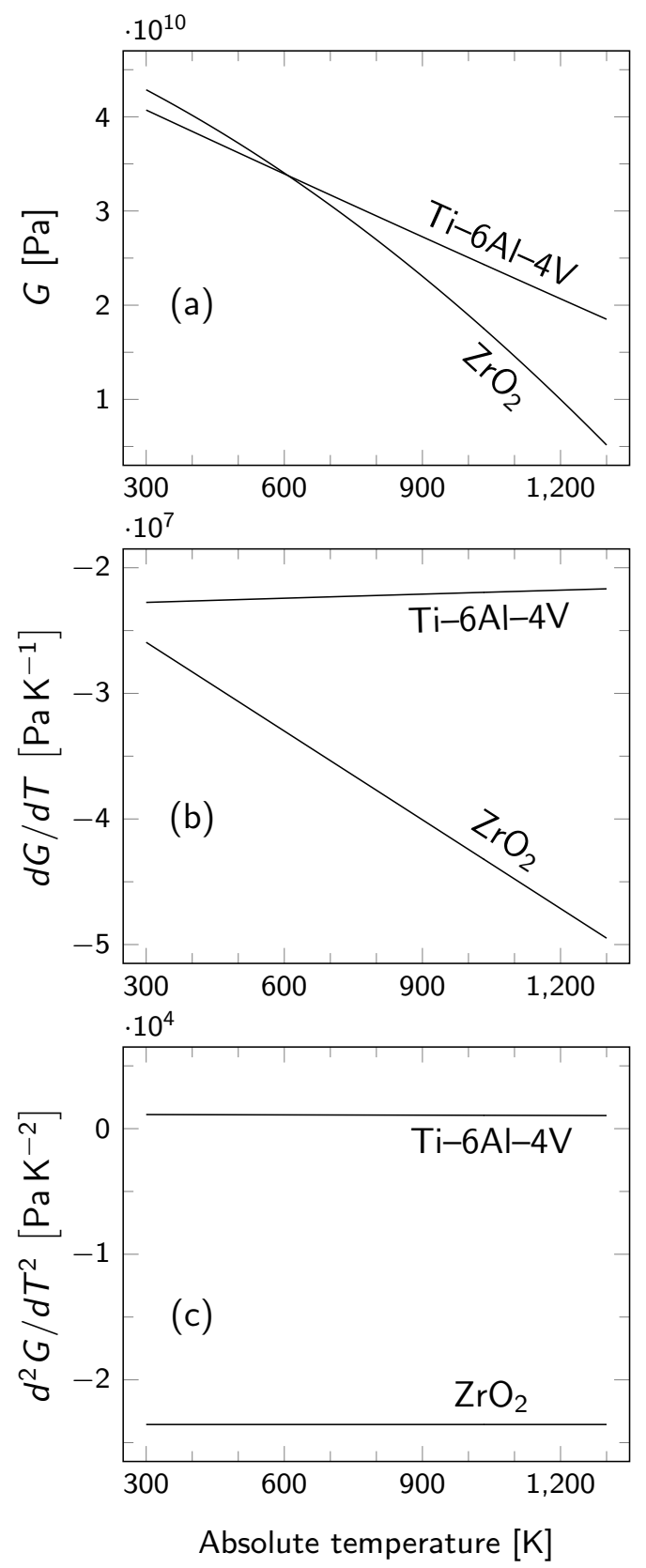

Figure 1: Constituent shear moduli (a) and their respective first (b) and second (c) $T$-derivatives as functions of temperature. 

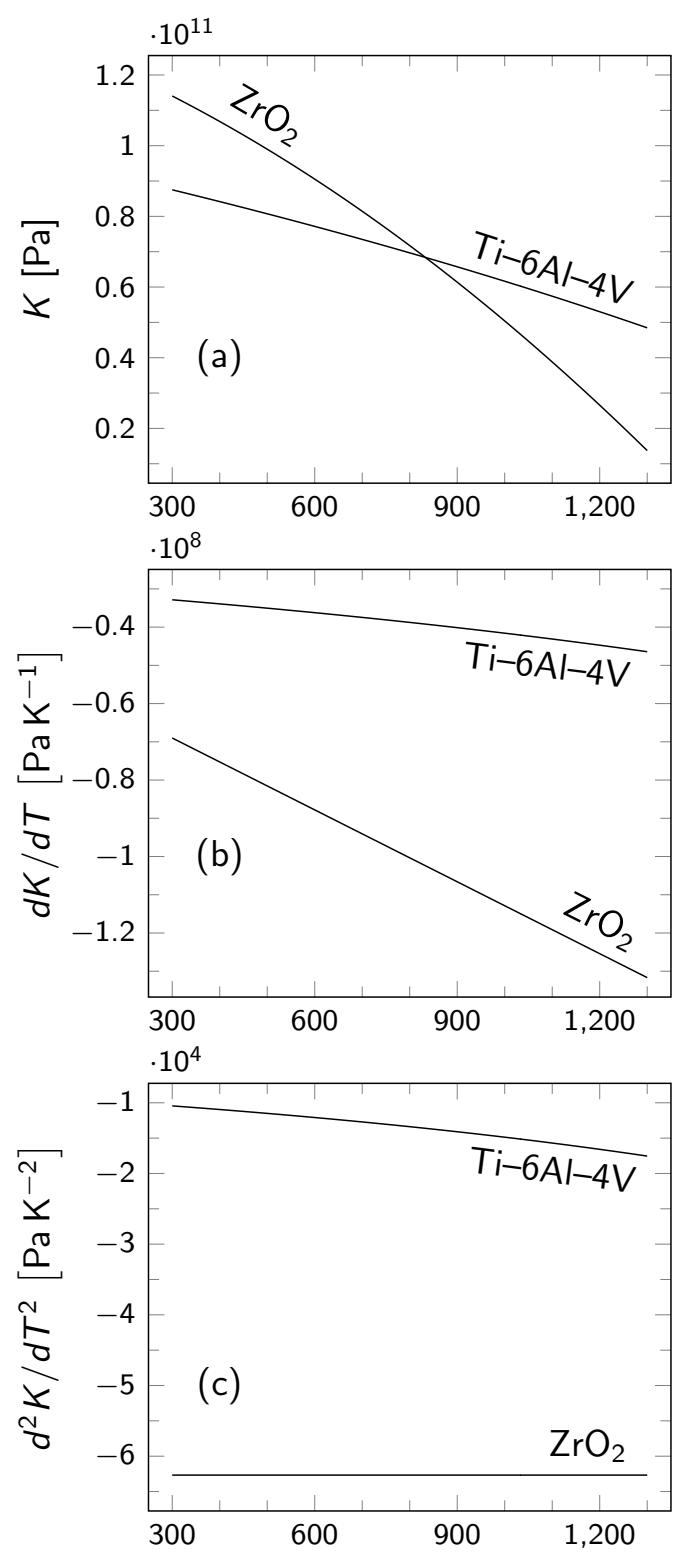

Absolute temperature $[\mathrm{K}]$

Figure 2: Constituent bulk moduli (a) and their respective first (b) and second (c) $T$-derivatives as functions of temperature. 

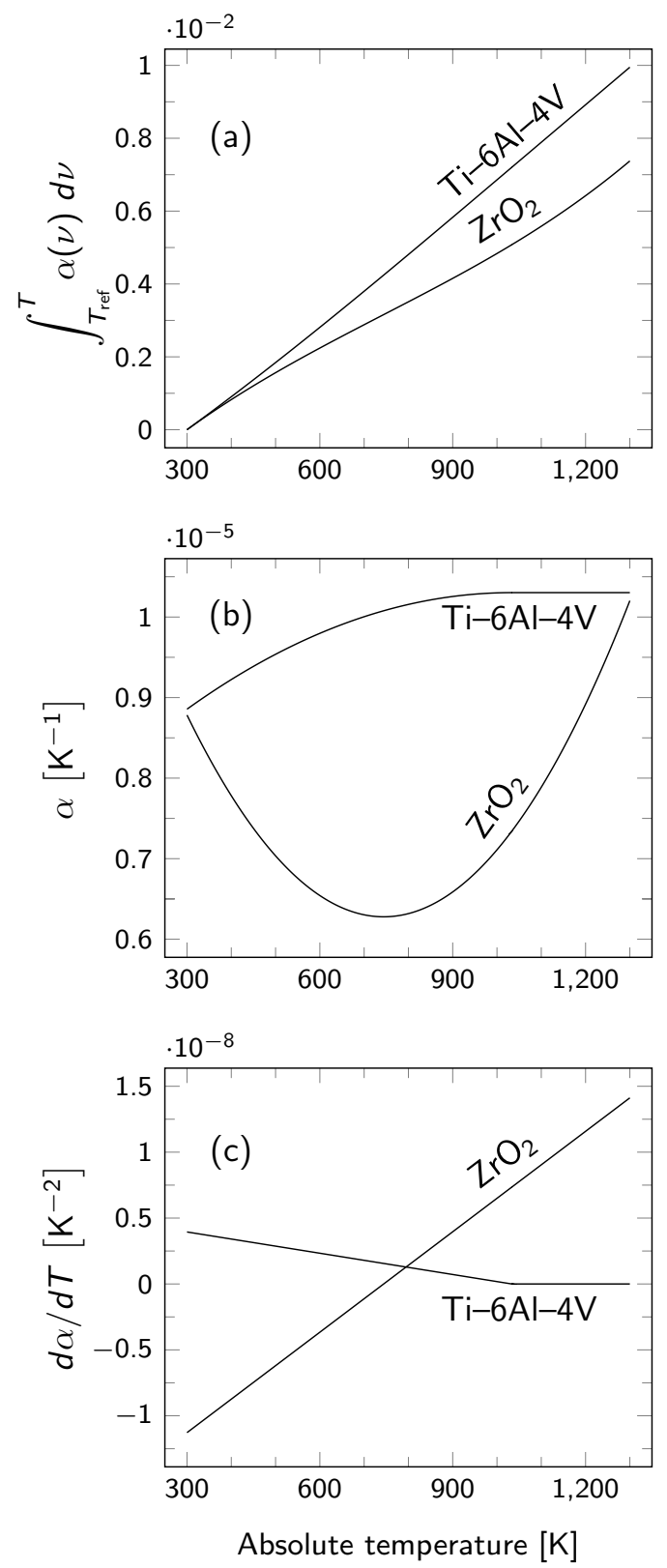

Figure 3: Constituent coefficients of free linear thermal expansion (b), their respective integrals from $T_{\text {ref }}$ (i.e., the constituent free linear thermal expansions from $T_{\text {ref }}$ ) (a) and their respective $T$-derivatives (c) as functions of temperature. 


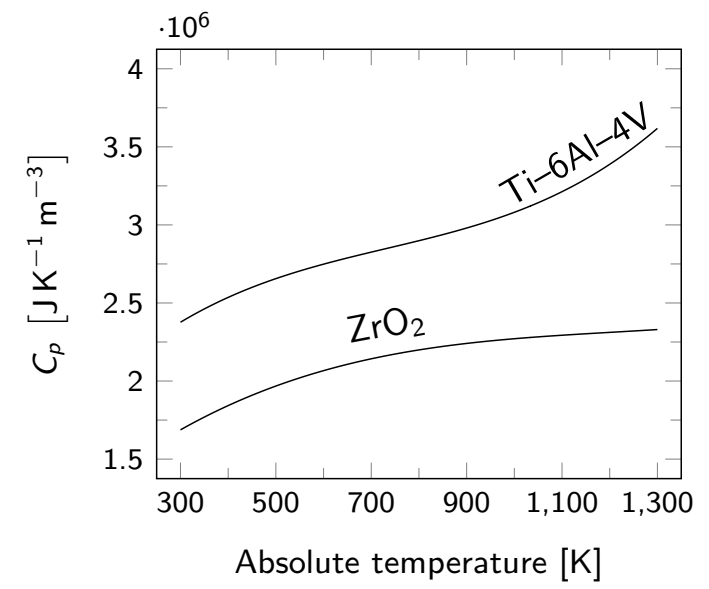

Figure 4: Constituent heat capacities at constant pressure per unit reference volume as functions of temperature. 

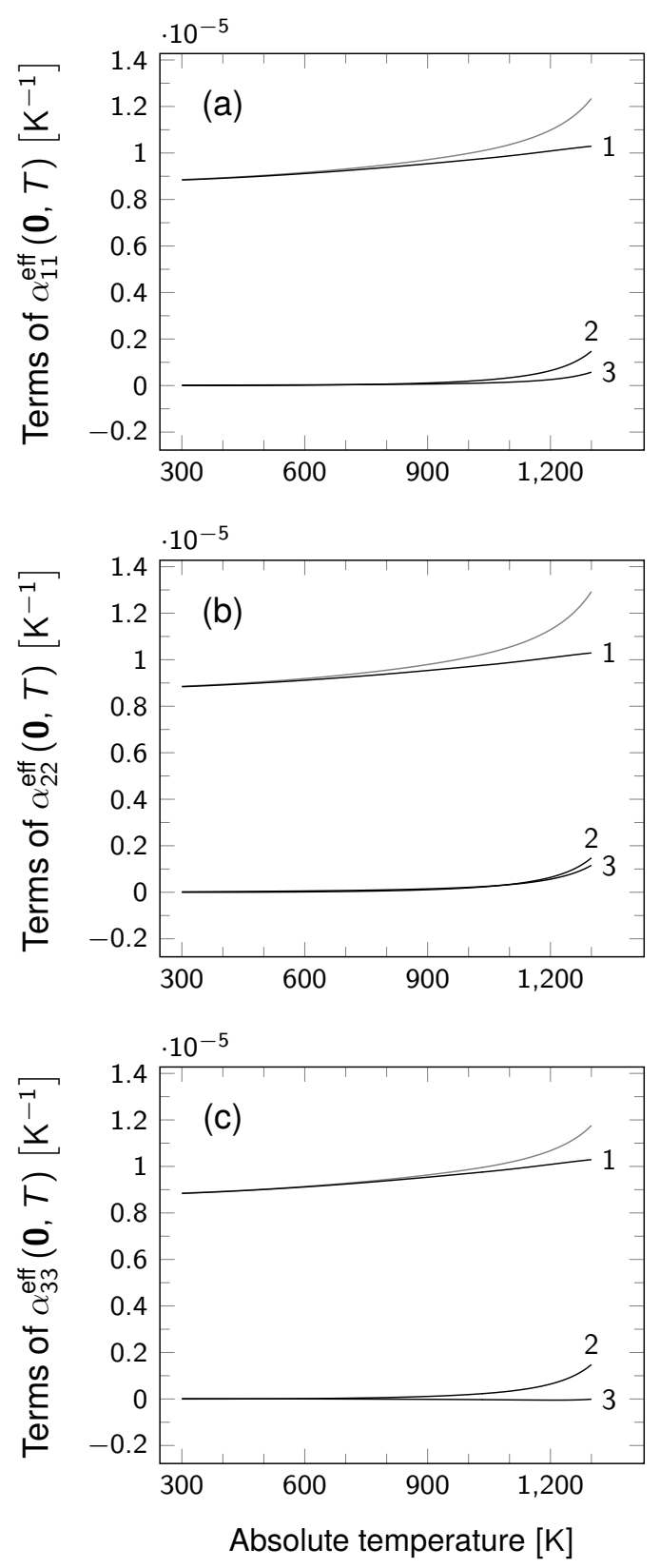

Figure 5: Decomposition of the non-zero components of the effective CFTE tensor in the presence of a superimposed eigenstrain field $\boldsymbol{m}_{*}$ such that $\boldsymbol{m}_{*}^{(\mathrm{m})}-$ $\boldsymbol{m}_{*}^{(\mathrm{i})}=10^{-3}\left(\boldsymbol{e}_{1} \otimes \boldsymbol{e}_{1}+2 \boldsymbol{e}_{2} \otimes \boldsymbol{e}_{2}\right)$. The curves labeled 1 correspond to Term 1 (i.e., $\left\langle\boldsymbol{B}^{\top} \boldsymbol{\alpha}\right\rangle$ ); the curves labeled 2, to Term 2 (i.e., $\left\langle(d \boldsymbol{B} / d T)^{\top} \boldsymbol{m}_{\mathrm{th}}\right\rangle$ ); the curves labeled 3, to Term 3 (i.e., $\left.\left\langle(d \boldsymbol{B} / d T)^{\top} \boldsymbol{m}_{*}\right\rangle\right)$. The gray curves correspond to the sum of the three terms, that is, to the non-zero components of $\boldsymbol{\alpha}^{\mathrm{eff}}(\mathbf{0}, T)$. 

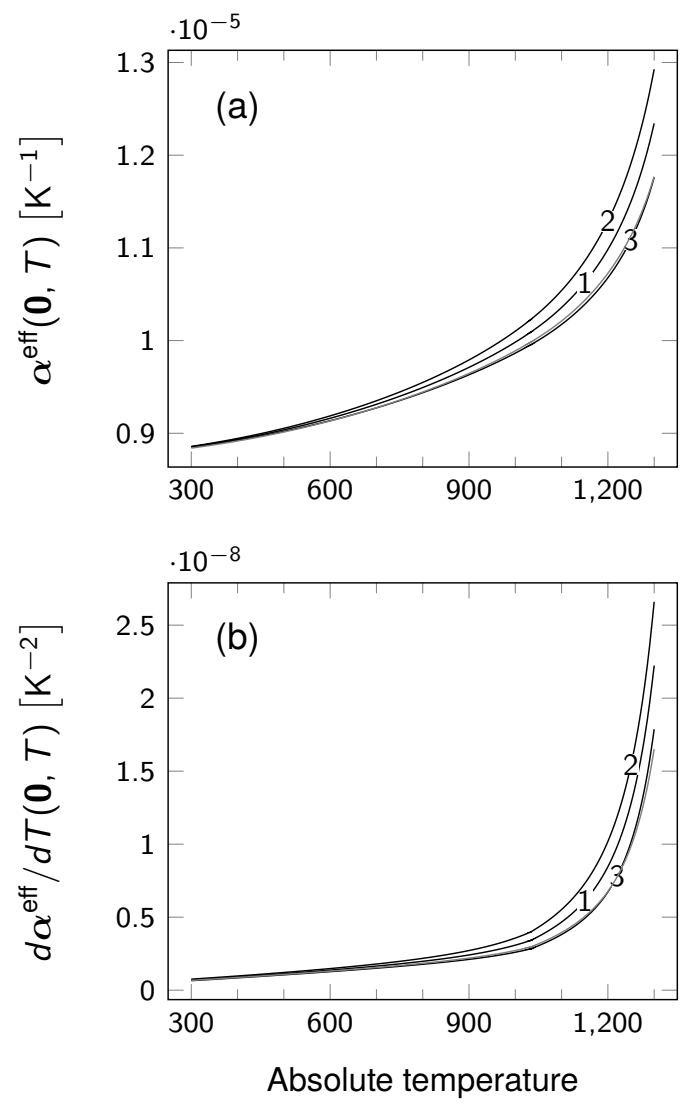

Figure 6: Non-zero components (a) and their $T$-derivatives (b) as functions of temperature for the effective CFTE tensor in the presence of a superimposed eigenstrain field $\boldsymbol{m}_{*}$ such that $\boldsymbol{m}_{*}^{(\mathrm{m})}-\boldsymbol{m}_{*}^{(\mathrm{i})}=10^{-3}\left(\boldsymbol{e}_{1} \otimes \boldsymbol{e}_{1}+2 \boldsymbol{e}_{2} \otimes \boldsymbol{e}_{2}\right)$. The curves labeled 1 (respectively 2,3) represent component $\alpha_{11}^{\mathrm{eff}}(\mathbf{0}, T)$ (a) and component $d \alpha_{11}^{\text {eff }} / d T(\mathbf{0}, T)$ (b) (respectively $\alpha_{22}^{\text {eff }}(\mathbf{0}, T)$ (a) and $d \alpha_{22}^{\text {eff }} / d T(\mathbf{0}, T)(\mathrm{b})$, $\alpha_{33}^{\text {eff }}(\mathbf{0}, T)(\mathrm{a})$ and $\left.d \alpha_{33}^{\text {eff }} / d T(\mathbf{0}, T)(\mathrm{b})\right)$ of $\boldsymbol{\alpha}^{\text {eff }}(\mathbf{0}, T)$. The gray curves, which are hardly distinguishable from the curves labeled 3 , correspond to the value of the coefficient of free thermal expansion (a) and that of its $T$-derivative in the case of no superimposed eigenstrain. 


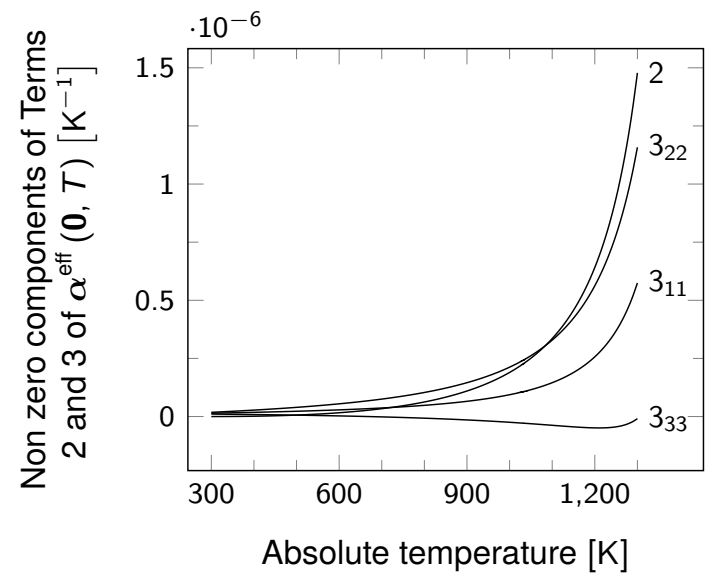

Figure 7: Comparison of the non zero components of Term 2 (i.e., $\left.\left\langle(d \boldsymbol{B} / d T)^{\top} \boldsymbol{m}_{\mathrm{th}}\right\rangle\right)$ and Term 3 (i.e., $\left.\left\langle(d \boldsymbol{B} / d T)^{\top} \boldsymbol{m}_{*}\right\rangle\right)$ of the effective CFTE tensor in the presence of a superimposed eigenstrain field $\boldsymbol{m}_{*}$ such that $\boldsymbol{m}_{*}^{(\mathrm{m})}-\boldsymbol{m}_{*}^{(\mathrm{i})}=10^{-3}\left(\boldsymbol{e}_{1} \otimes \boldsymbol{e}_{1}+2 \boldsymbol{e}_{2} \otimes \boldsymbol{e}_{2}\right)$. The curve labeled 2 represents the diagonal term of Term 2, which is isotropic. The curve labeled $3_{11}$ represents component 11 of Term 3, and so on. The components of Term 2 are zero at $300 \mathrm{~K}$. Those of Term 3 are relatively small (around $10^{-8}$ ) but non-zero at $300 \mathrm{~K}$. 

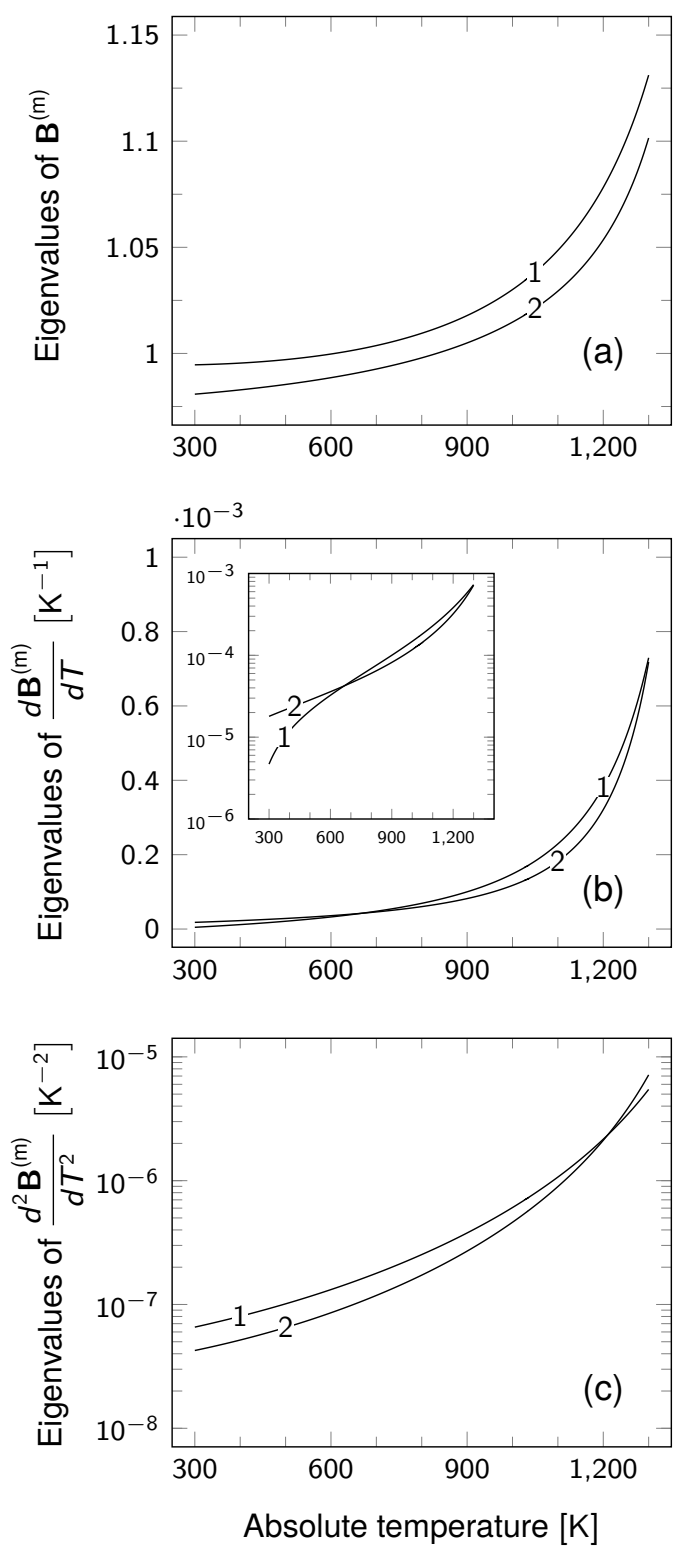

Figure 8: Eigenvalues of $\boldsymbol{B}^{(\mathrm{m})}$ (a) and their first (b) and second (c) T-derivatives as functions of temperature. The curves labeled 1 (respectively 2) correspond to the eigenvalue of multiplicity 5 (respectively 1). A linear scale is used for the ordinate axes of subfigures (a) and (b); a log scale, for the ordinate axes of subfigure (c) and the inset in subfigure (b). 

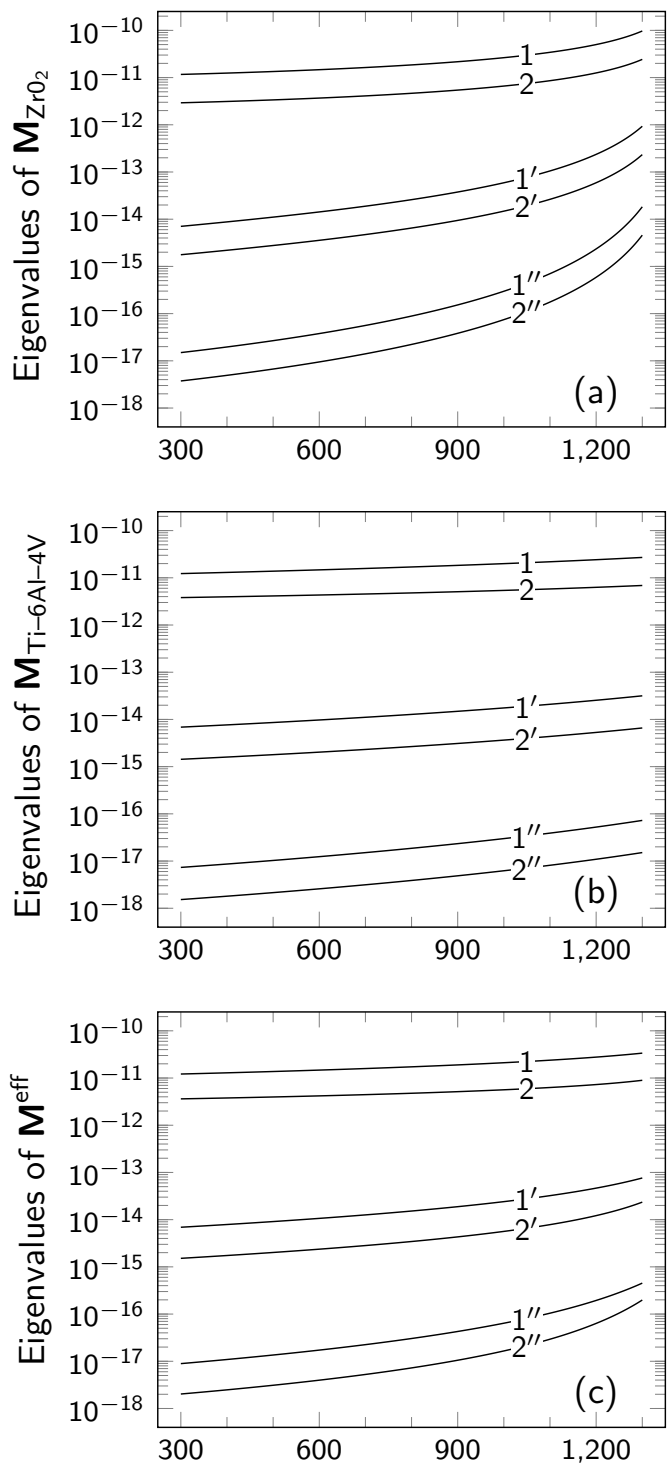

Absolute temperature $[\mathrm{K}]$

Figure 9: Eigenvalues of the compliance tensors (curves labeled 1 and 2), those of their respective first (curves labeled $1^{\prime}$ and $2^{\prime}$ ) and second (curves labeled $1^{\prime \prime}$ and $2^{\prime \prime}$ ) T-derivatives as functions of temperature for $\mathrm{ZrO}_{2}$ (a), Ti6Al4V (b), and the composite (c). The curves labeled $1,1^{\prime}$, and $1^{\prime \prime}$ correspond to the eigenvalues of multiplicity 5 . 

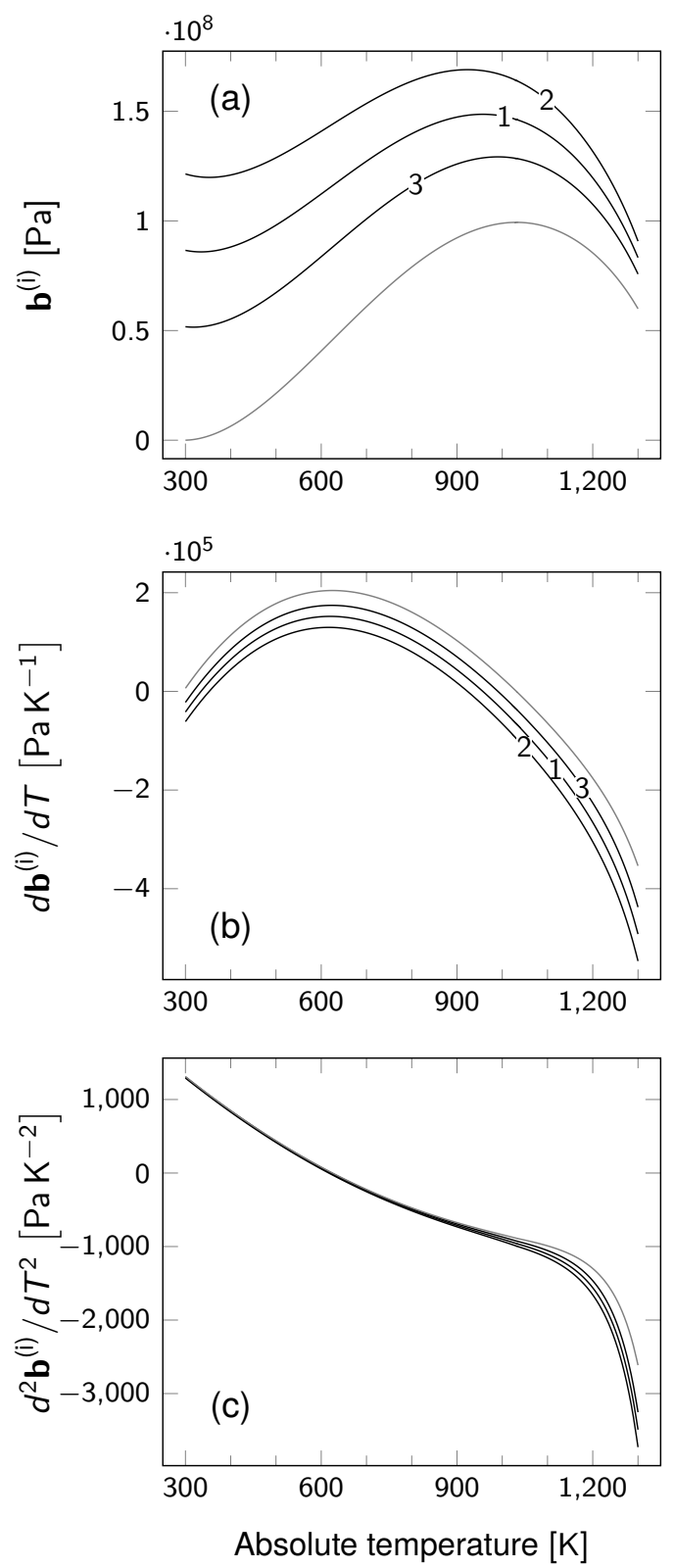

Figure 10: The three non vanishing components (a) and their first (b) and second (c) $T$-derivatives as functions of temperature for $\boldsymbol{b}^{(\mathrm{i})}$ in the presence of a superimposed eigenstrain field $\boldsymbol{m}_{*}$ such that $\boldsymbol{m}_{*}^{(\mathrm{m})}-\boldsymbol{m}_{*}^{(\mathrm{i})}=10^{-3}\left(\boldsymbol{e}_{1} \otimes \boldsymbol{e}_{1}+2 \boldsymbol{e}_{2} \otimes \boldsymbol{e}_{2}\right)$. The curves labeled 1 (respectively 2,3 ) represent components 11 (respectively 22,33 ). The gray curves correspond to the case of no superimposed eigenstrain field, in which case $\boldsymbol{b}^{(\mathrm{i})}$ and its first and second $T$-derivatives are isotropic. In subfigure (c), the black curves are ordered as in subfigure (b). 


\section{List of Tables}

1 Material properties of the constituents as functions of the absolute temperature (Tanigawa et al., 1997). Heat capacity refers to the heat capacity per unit reference volume at constant pressure. The quantity $\alpha$ entering the expression for the mass density of a material is its linear CTE. . . . . . . . . . . . . . . 


\begin{tabular}{ll}
\hline Property & Expression \\
\hline $\mathrm{ZrO}_{2}$ & \\
Heat capacity $[\mathrm{J} /(\mathrm{kgK})]$ & $2.74 \cdot 10^{2}+7.95 \cdot 10^{-1} T-6.19 \cdot 10^{-4} T^{2}+1.71 \cdot 10^{-7} T^{3}$ \\
$\quad$ Linear CTE $[1 / \mathrm{K}]$ & $13.31 \cdot 10^{-6}-18.9 \cdot 10^{-9} T+12.7 \cdot 10^{-12} T^{2}$ \\
$\quad$ Mass density $\left[\mathrm{kg} / \mathrm{m}^{3}\right]$ & $3657.0 /(1+\alpha(T-300))^{3}$ \\
$\quad$ Young's modulus $[\mathrm{GPa}]$ & $132.2-50.3 \cdot 10^{-3} T-31.4 \cdot 10^{-6} T^{2}$ \\
$\quad$ Poisson's ratio & 0.333 \\
$\mathrm{Ti}-6 \mathrm{Al}-4 \mathrm{~V}$ & \\
Heat capacity $[\mathrm{J} /(\mathrm{kgK})]$ & $3.5 \cdot 10^{2}+8.78 \cdot 10^{-1} T-9.74 \cdot 10^{-4} T^{2}+4.43 \cdot 10^{-7} T^{3}$ \\
Linear CTE $[1 / \mathrm{K}]$ & $7.43 \cdot 10^{-6}+5.56 \cdot 10^{-9} T-2.69 \cdot 10^{-12} T^{2}$ for $300 \mathrm{~K} \leq T \leq 1100 \mathrm{~K}$ \\
& $10.291 \cdot 10^{-6}$ for $1100 \mathrm{~K} \leq T \leq 1300 \mathrm{~K}$ \\
Mass density $\left[\mathrm{kg} / \mathrm{m}^{3}\right]$ & $4420.0 /(1+\alpha(T-300))^{3}$ \\
$\quad$ Young's modulus $[\mathrm{GPa}]$ & $122.7-0.0565 T$ \\
Poisson's ratio & $0.289+32.0 \cdot 10^{-6} T$ \\
\hline
\end{tabular}

Table 1: Material properties of the constituents as functions of the absolute temperature (Tanigawa et al., 1997). Heat capacity refers to the heat capacity per unit reference volume at constant pressure. The quantity $\alpha$ entering the expression for the mass density of a material is its linear CTE. 